\title{
Phosphorylation state-dependent modulation of spinal glycine receptors alleviates inflammatory pain
}

\author{
Mario A. Acuña, ${ }^{1,2}$ Gonzalo E. Yévenes, ${ }^{1,3}$ William T. Ralvenius, ${ }^{1,2}$ Dietmar Benke, ${ }^{1,2,4}$ Alessandra Di Lio, ${ }^{1,2}$ Cesar O. Lara, ${ }^{3}$ \\ Braulio Muñoz, ${ }^{3}$ Carlos F. Burgos, ${ }^{3}$ Gustavo Moraga-Cid, ${ }^{3,5}$ Pierre-Jean Corringer, ${ }^{5}$ and Hanns Ulrich Zeilhofer ${ }^{1,2,4,6}$ \\ Institute of Pharmacology and Toxicology, University of Zurich, Zurich, Switzerland. ${ }^{2}$ Neuroscience Center Zurich, Zurich, Switzerland. ${ }^{3}$ Department of Physiology, Faculty of Biological Sciences, \\ University of Concepcion, Concepcion, Chile. ${ }^{4}$ Drug Discovery Network Zurich, Zurich, Switzerland. ${ }^{5}$ Channel-Receptors Unit, Institute Pasteur, Paris, France. \\ ${ }^{6}$ Institute of Pharmaceutical Sciences, Swiss Federal Institute of Technology Zurich, Zurich, Switzerland.
}

\begin{abstract}
Diminished inhibitory neurotransmission in the superficial dorsal horn of the spinal cord is thought to contribute to chronic pain. In inflammatory pain, reductions in synaptic inhibition occur partially through prostaglandin $E_{2}-\left(P C E_{2}-\right)$ and PKA-dependent phosphorylation of a specific subtype of glycine receptors (GlyRs) that contain $\alpha 3$ subunits. Here, we demonstrated that 2,6-di-tert-butylphenol (2,6-DTBP), a nonanesthetic propofol derivative, reverses inflammation-mediated disinhibition through a specific interaction with heteromeric $\alpha \beta$ GlyRs containing phosphorylated $\alpha 3$ subunits. We expressed mutant GlyRs in HEK293T cells, and electrophysiological analyses of these receptors showed that 2,6-DTBP interacted with a conserved phenylalanine residue in the membrane-associated stretch between transmembrane regions 3 and 4 of the GlyR $\alpha 3$ subunit. In native murine spinal cord tissue, 2,6-DTBP modulated synaptic, presumably $\alpha \beta$ heteromeric, GlyRs only after priming with $\mathrm{PGE}_{2}$. This observation is consistent with results obtained from molecular modeling of the $\alpha-\beta$ subunit interface and suggests that in $\alpha 3 \beta \mathrm{GlyRs}$, the binding site is accessible to 2,6-DTBP only after PKA-dependent phosphorylation. In murine models of inflammatory pain, 2,6-DTBP reduced inflammatory hyperalgesia in an $\alpha 3$ GlyR-dependent manner. Together, our data thus establish that selective potentiation of GlyR function is a promising strategy against chronic inflammatory pain and that, to our knowledge, 2,6-DTBP has a unique pharmacological profile that favors an interaction with GlyRs that have been primed by peripheral inflammation.
\end{abstract}

\section{Introduction}

Chronic pain states are associated with complex molecular and cellular changes in the peripheral and central nervous system (1-3). An increasing body of evidence indicates that diminished inhibitory GABAergic and glycinergic neurotransmission at the level of the spinal dorsal horn makes an important contribution to chronic pain states $(2,4,5)$. A variety of mechanisms have been identified that differentially contribute to diminished synaptic inhibition in various inflammatory and neuropathic pain states $(1,2)$. In the case of inflammatory pain, a reduction in glycinergic inhibition occurring through $\mathrm{PGE}_{2}$ - and PKA-dependent phosphorylation of a specific subtype of glycine receptors (GlyRs; i.e., those containing the $\alpha 3$ subunit $[\alpha 3 G l y R]$ ) has a major role (6). Restoring the activity of the synaptic $\alpha 3$ GlyR through positive allosteric modulators may therefore constitute a mechanism-based therapeutic approach against chronic inflammatory pain.

Despite the importance of GlyRs in a variety of physiological processes, GlyR pharmacology is still poorly developed, and only a few GlyR modulators are available at present (7-9). Some of these compounds, such as several propofol derivatives, modulate GlyRs at nanomolar or even subnanomolar concentrations $(10,11)$, but none of them are fully specific for GlyRs and many of them also

Authorship note: M.A. Acuña and G.E. Yévenes contributed equally to this work. Conflict of interest: The authors have declared that no conflict of interest exists. Submitted: July 17, 2015; Accepted: April 14, 2016.

Reference information: J Clin Invest. 2016;126(7):2547-2560. doi:10.1172/JCI83817. modulate the function of other ion channels (8). This lack of specificity has hampered establishing causal links between molecular and therapeutic actions (12-15).

Using molecular, electrophysiological, and behavioral approaches, we explored the basis of GlyR allosteric modulation by the nonanesthetic propofol derivative 2,6-di-tert-butylphenol (2,6-DTBP). 2,6-DTBP potentiates currents through recombinant $\alpha 1$ GlyRs (16) but has negligible activity at $\mathrm{GABA}_{\mathrm{A}}$ receptors $\left(\right.$ GABA $\left._{A} \mathrm{Rs}\right)(17,18)$. Here, we show that 2,6 -DTBP is a positive $\alpha 3$ GlyR allosteric modulator that effectively restores the loss of a3GlyR-mediated inhibition in inflammatory pain states, at least partially through a selective potentiation of phosphorylated GlyRs containing $\alpha 3$ and $\beta$ subunits. A specific phenylalanine residue in the $\alpha 3$ subunit appears to be critical for this potentiation. Thus, our results establish a potentially novel mechanism for pharmacological intervention against inflammatory pain through phosphorylation-specific potentiation of the spinal $\alpha 3 \mathrm{GlyR}$.

\section{Results}

Conformation-specific potentiation of recombinant $\alpha 3 G l y R$ by 2,6$D T B P$. We first assessed the effects of 2,6-DTBP on glycine-evoked currents through recombinant GlyRs expressed in HEK293T cells (for glycine response parameters of all GlyR variants tested in this study, see Supplemental Table 1; supplemental material available online with this article; doi:10.1172/JCI83817DS1). Homopentameric $\alpha 3$ GlyRs were strongly potentiated by micromolar concentrations of 2,6-DTBP (Figure 1, A and B). The efficacy of 
A
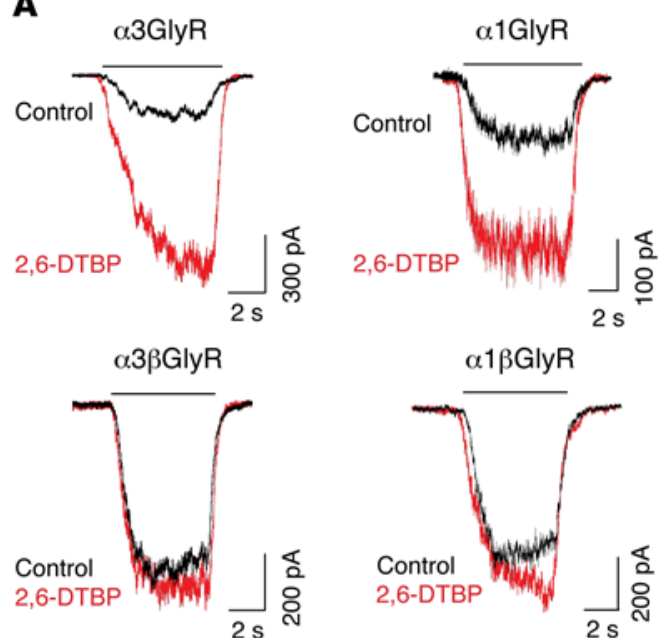

C

$\alpha 1 \beta$ GlyR

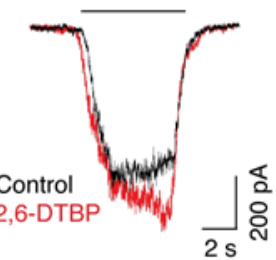

B

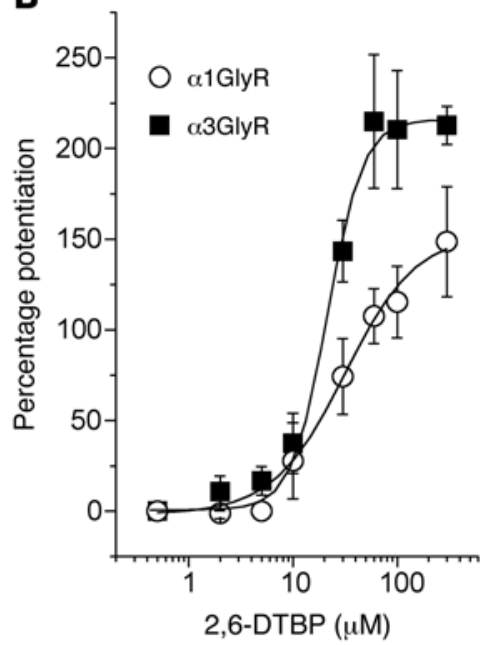

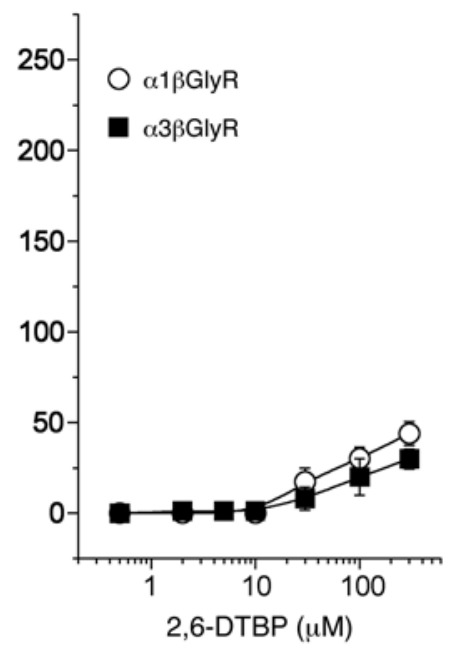

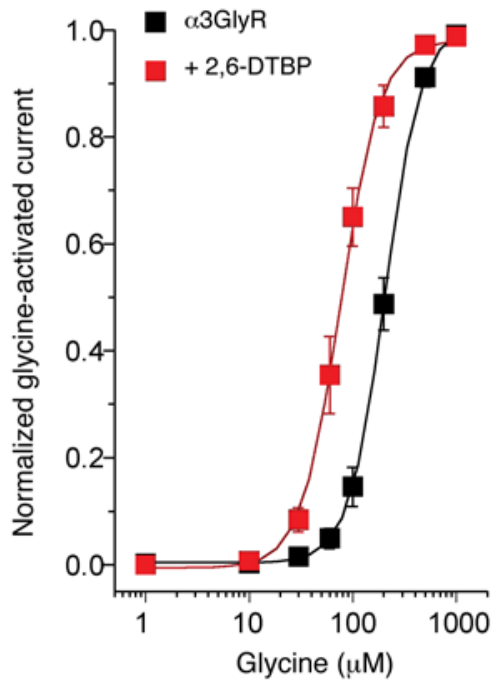

$\mathbf{E}$

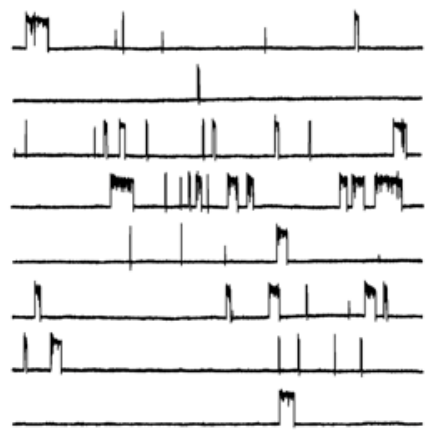

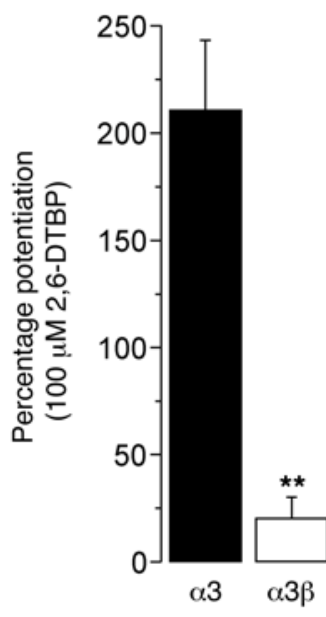
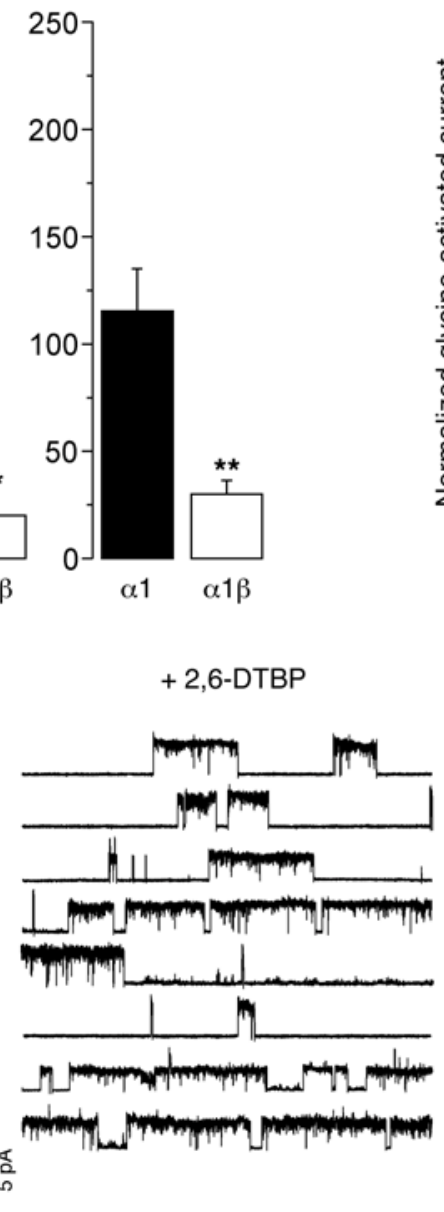

$\mathbf{F}$

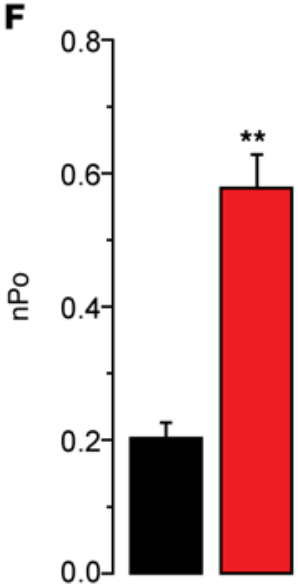

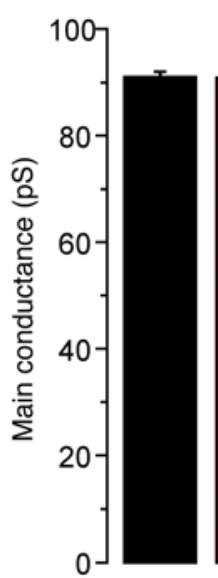

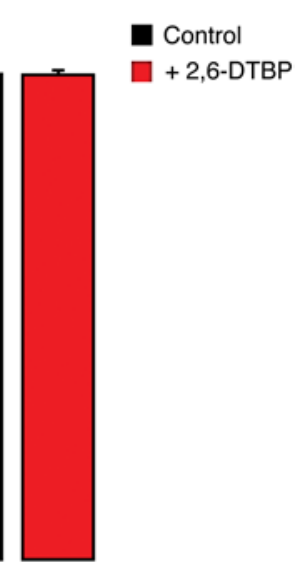

Figure 1. Modulation of recombinant GlyRs by 2,6-DTBP in HEK293T cells. (A) Example whole-cell current traces evoked by glycine (EC $\left.{ }_{10}\right)$ in the absence or presence of 2,6-DTBP $(100 \mu \mathrm{M})$ in homomeric $\alpha 3$ or $\alpha 1 \mathrm{ClyRs}$, and in heteromeric $\alpha 3 \beta$ or $\alpha 1 \beta \mathrm{GlyRs}$. (B) Concentration-response curves of 2,6-DTBP obtained with an $\mathrm{EC}_{10}$ of glycine in homomeric $\alpha 3$ or $\alpha 1 \mathrm{ClyRs}$, and in heteromeric $\alpha 3 \beta$ or $\alpha 1 \beta \mathrm{GlyRs}$. (C) Heteromeric $\alpha 3 / \beta$ and $\alpha 1 / \beta \mathrm{GlyRs}$ are significantly less susceptible to modulation by 2,6-DTBP than homomeric $\alpha 1$ and $\alpha 3$ GlyRs. ${ }^{* *} P<0.01$, unpaired $t$ test. (D) Concentration-response curves of glycine for the homomeric $\alpha 3 G$ lyR in the absence or in the presence of 2,6-DTBP $(100 \mu \mathrm{M}$, red). (E) Single-channel current traces recorded from membranes expressing $\alpha 3 \mathrm{ClyRs}$ in the presence and the absence of 2,6-DTBP $(10 \mu \mathrm{M})$. (F) 2,6-DTBP increases ion channel open probability (nPo) but not single-channel main conductance. ${ }^{*} P<0.01$, paired $t$ test. Data are the mean \pm SEM from 6 to 10 cells $(\mathbf{B}-\mathbf{D})$ or 6 patches per group (F). 
A

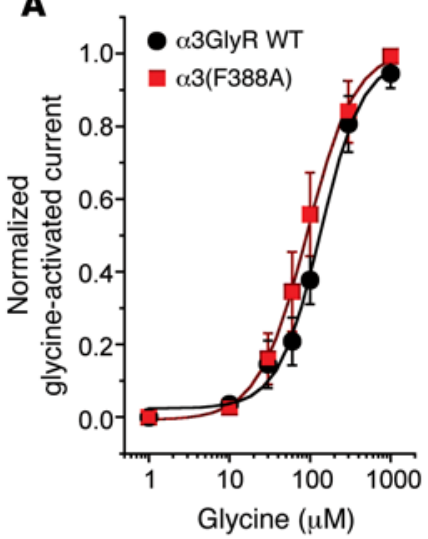

D

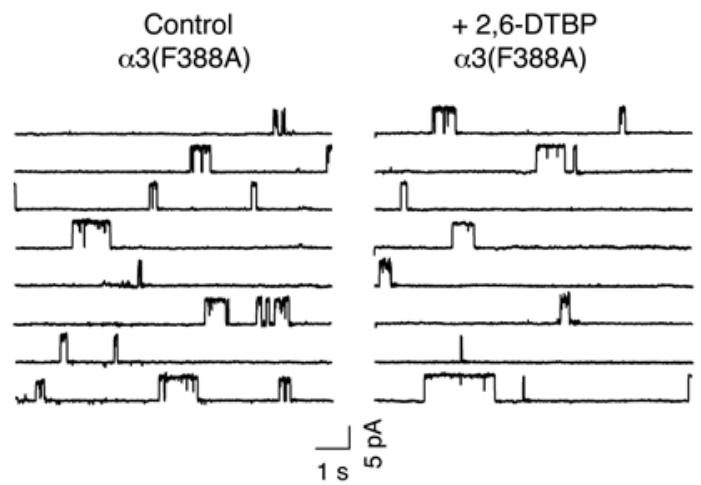

B
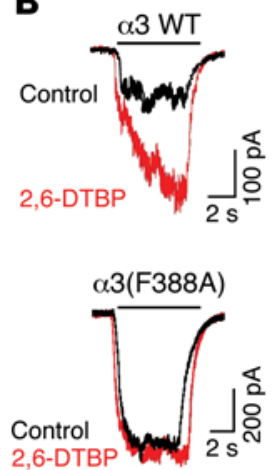

C

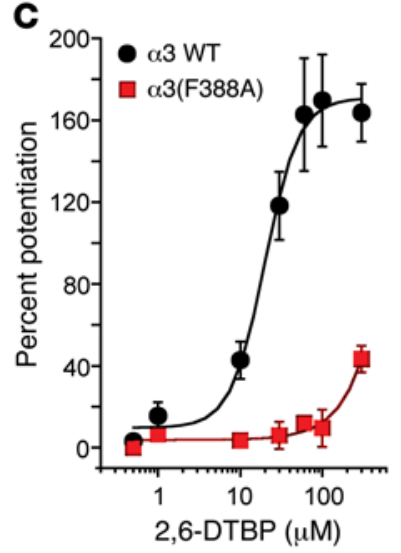

E

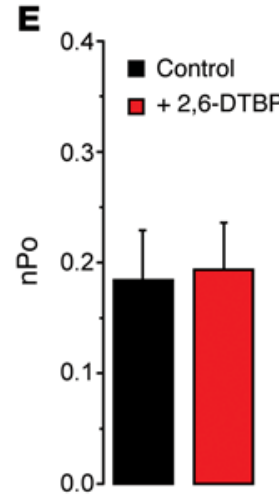

$\mathbf{F}$

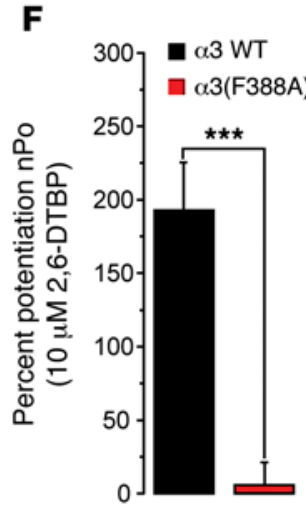

Figure 2. A molecular site for the actions of 2,6-DTBP on $\mathbf{\alpha 3 G l y R s . ~ ( A ) ~ C o n c e n t r a t i o n - r e s p o n s e ~ c u r v e s ~ o f ~ g l y c i n e ~ i n ~ w i l d - t y p e ~ a n d ~ ( F 3 8 8 A ) ~ p o i n t - m u t a t e d ~}$ $\alpha 3 G$ lyRs. (B) Traces of glycine-activated whole-cell currents recorded from cells expressing wild-type or (F388A) point-mutated $\alpha 3 G$ lyRs in the absence or presence of 2,6-DTBP $(100 \mu \mathrm{M})$. (C) Concentration-response curves of 2,6-DTBP for wild-type and (F388A) point-mutated $\alpha 3$ ClyRs. (D) Examples of single-channel recordings from membranes expressing point-mutated (F388A) $\alpha 3 \mathrm{GlyRs}$ in the presence or absence of 2,6-DTBP. (E) lon channel nPo of the (F388A) $\alpha 3$ GlyR in the absence or presence of 2,6-DTBP $(10 \mu \mathrm{M})$. (F) Percentage change of nPo following the application of 2,6-DTBP in wild-type and (F388A) point-mutated $\alpha 3$ GlyRs. ${ }^{* *} P<0.001$, unpaired $t$ test. All data are the mean \pm SEM from 6 to 9 cells per group.

potentiation by 2,6-DTBP (determined at a 2,6-DTBP concentration of $100 \mu \mathrm{M}$ and at the $\mathrm{EC}_{10}$ of glycine) was higher for $\alpha 3 \mathrm{GlyRs}$ than for $\alpha 1$ GlyRs $(211 \% \pm 30 \%$ versus $115 \% \pm 19 \%, P<0.05, n=6$, unpaired $t$ test). We next assessed the modulation of heteromeric $\alpha \beta G l y R s$ (as these receptors constitute most GlyRs at postsynaptic sites). The efficient expression of heteromeric GlyRs containing both $\alpha$ and $\beta$ subunits was confirmed by their relative insensitivity to picrotoxin (PTX), which blocks homomeric GlyRs with higher potency than $\alpha \beta$ heteromeric receptors (Supplemental Figure 1). Compared with homomeric GlyRs, potentiation of heteromeric $\alpha 3 \beta$ or $\alpha 1 \beta$ GlyRs was significantly weaker $(20 \% \pm 10 \%$ and $30 \%$ $\pm 6 \%$ for $\alpha 3 \beta$ and $\alpha 1 \beta$ GlyRs, respectively, Figure $\left.1, A^{-} \mathrm{C}\right)$. We then evaluated the biophysical mechanisms underlying the allosteric modulation by 2,6-DTBP of a3GlyRs. Whole-cell recordings showed that 2,6-DTBP caused a leftward shift in the glycine concentration-response curve of the $\alpha 3 \mathrm{GlyR}$. The $\mathrm{EC}_{50}$ values were $206 \pm 1.0 \mu \mathrm{M}$ and $76 \pm 2.3 \mu \mathrm{M}$, under control conditions and in the presence of $100 \mu \mathrm{M} 2,6$-DTBP, respectively $(P<0.001$, unpaired $t$ test) (Figure 1D), suggesting an increase in the apparent affinity of the receptor for its agonist. 2,6-DTBP did not affect the maximal current amplitude elicited by $1 \mathrm{mM}$ glycine $(5.0 \pm 1.0 \mathrm{nA}$ versus $4.8 \pm 1.1 \mathrm{nA}$, in the presence and absence of $100 \mu \mathrm{M} 2,6-\mathrm{DTBP}$, respectively). Single-channel recordings in the cell-attached con- figuration (19) showed that 2,6-DTBP significantly increased the ion channel open probability $(\mathrm{nPo})$ (control $\mathrm{nPo}=0.20 \pm 0.02$ versus 2,6-DTBP nPo $=0.58 \pm 0.10$, paired $t$ test, $P<0.01$, Figure 1, E and F, and Supplemental Table 2) without apparent changes in the main conductance levels (control $\gamma=91.0 \pm 1.20 \mathrm{pS}$ versus 2,6-DTBP $\gamma=90.1 \pm 0.92 \mathrm{pS}$, paired $t$ test, Figure $1, \mathrm{E}$ and F, and Supplemental Table 2).

In order to explore the molecular determinants of $\alpha 3 \mathrm{GlyR}$ modulation by 2,6-DTBP, we first focused on molecular sites in $\alpha 1$ GlyR and $\alpha 3$ GlyR involved in the modulation by propofol, the parent compound of 2,6-DTBP. Previous analyses of structureactivity relationships have identified S267 in TM2, A288 in TM3, and F380 in the interface between the intracellular region and the TM4 domain as critical for the modulation of the $\alpha 1$ GlyR by propofol (20-22). All three residues are conserved between $\alpha 1$ GlyR and $\alpha 3$ GlyR. Other studies have, however, found that mutation of two of these residues (S267 and A288) also severely interferes with GlyR function itself $(23,24)$. By contrast, mutation of F380 diminished propofol sensitivity without altering ion channel gating and conductance (21). We therefore examined whether the mutation of phenylalanine to alanine in position 388 (F388A) would affect the sensitivity of the $\alpha 3$ GlyR to 2,6-DTBP. Whole-cell recordings revealed that the F388A mutation did not change the sensitivity 
A
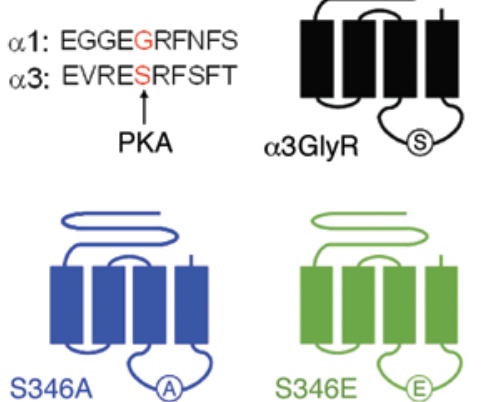

D $\alpha 3(\mathrm{~S} 346 \mathrm{~A}) \beta$
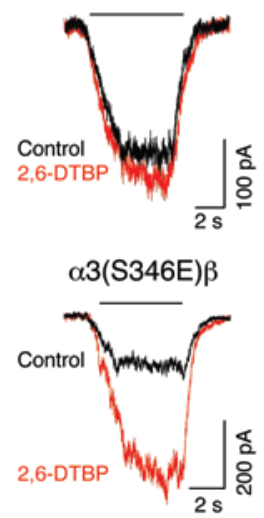

G

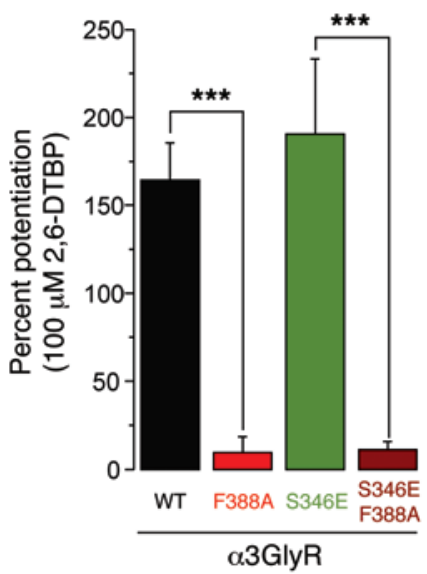

B
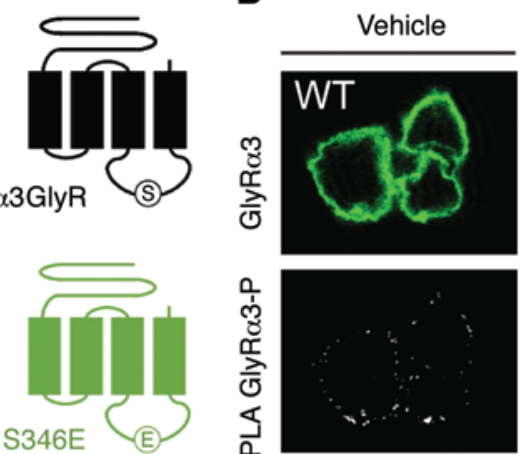

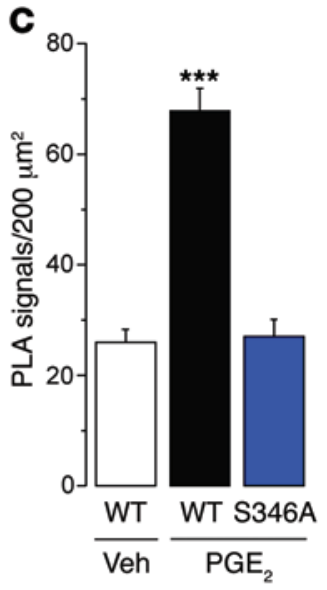

$\mathbf{F}$
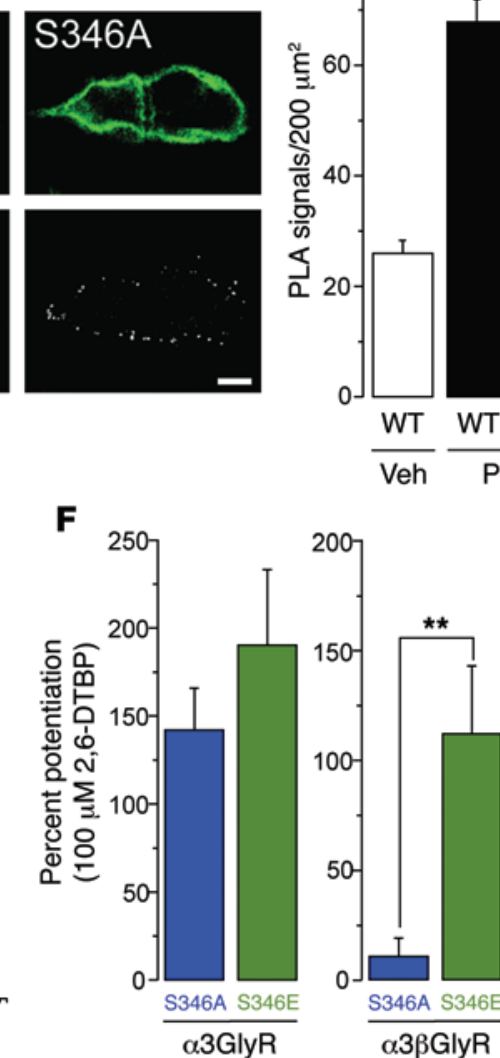
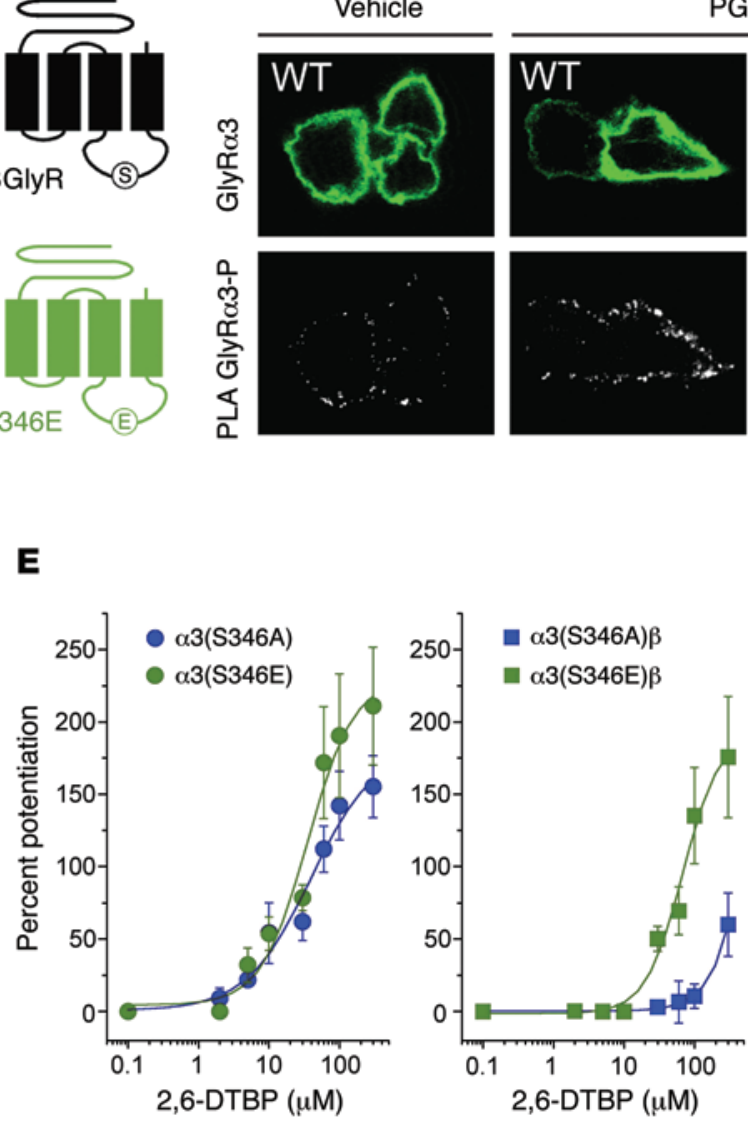

H
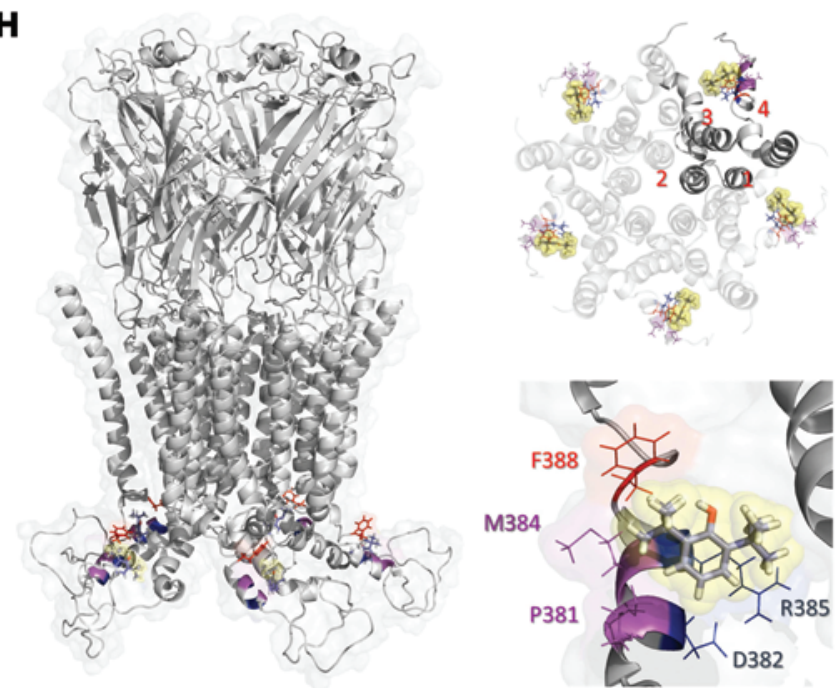

Figure 3. Influence of $\mathbf{S 3 4 6}$ phosphomutation in $\mathbf{\alpha 3 G l y R}$ on allosteric modulation by $\mathbf{2 , 6 - D T B P}$. (A) Localization of the S346 residue in the primary sequence of the mouse GlyR $\alpha 3$ subunit, the corresponding sequence in the GlyR $\alpha 1$ subunit, and schematic diagram illustrating this site in the $\alpha 3 G$ lyR topology. (B) Proximity ligation assay (PLA) demonstrating phosphorylation of $\alpha 3 G$ lyR by PGE $_{2}(1 \mu \mathrm{M})$ in HEK293T cells transfected with the EP2 receptor and wild-type GlyR $\alpha 3$ or (S346A) point-mutated GlyR $\alpha 3$ subunit plasmids. Top: $\alpha 3$ GlyR immunofluorescence, bottom: PLA signal. Scale bar: $5 \mu$ m. (C) PGE ${ }_{2}$ significantly increased PLA signals in HEK293T cells transfected with wild-type but not with (S346A) GlyR $\alpha 3$ subunits. ${ }^{* * *} P<0.001$, ANOVA followed by Bonferroni post-hoc test, $\mathrm{F}(2,141)=49.84 ; P<0.001$, $P \mathrm{PE}_{2}$ in wild-type $\alpha 3 G l y R$-transfected cells versus the other two conditions. (D) Example traces of glycine-activated currents in $\alpha 3$ (S346A) $\beta$ or $\alpha 3$ (S346E) $\beta$ GlyRs in the absence and presence of 2,6-DTBP (100 $\mu \mathrm{M})$. (E) Concentration-response curves for 2,6-DTBP in homomeric $\alpha 3$ (left) and heteromeric $\alpha 3 \beta$ GlyRs (right) containing S346E or S346A mutations. (F) The phosphomimicking S346E mutation restored 2,6-DTBP sensitivity of $\alpha 3 \beta$ GlyRs but had little effect on that of homomeric $\alpha 3$ GlyRs. ${ }^{*} P<0.01$, unpaired $t$ test. (G) The F388A point mutation in $\alpha 3 G$ lyRs abolished 2,6 -DTBP sensitivity. Introducing the S346E mutation did not restore 2,6-DTBP sensitivity in homomeric $\alpha 3$ (F388A)GlyRs or heteromeric $\alpha 3$ (F388A) $\beta$ GlyRs. ${ }^{* *} P<0.01,{ }^{* * *} P<$ 0.001 , ANOVA followed by Bonferroni post-hoc test, $F(3,35)=20.7$ (left) and $F(2,16)=8.81$ (right). (H) Structural model of the $\alpha 3 G$ lyR. Left: location of the F388 residue (red) and of 2,6-DTBP (yellow sphere) in a homopentameric $\alpha 3 G$ lyR. Other relevant residues are shown in magenta (P381 and M384) and blue (D382 and R385). Top, right: view from the middle of the plasma membrane on the putative acceptor sites with 2,6-DTBP bound (in yellow spheres) within a pentameric complex. Bottom, right: detailed view of the putative acceptor site of 2,6-DTBP. The hydrophobic interaction supported by F388 is highlighted in red. 

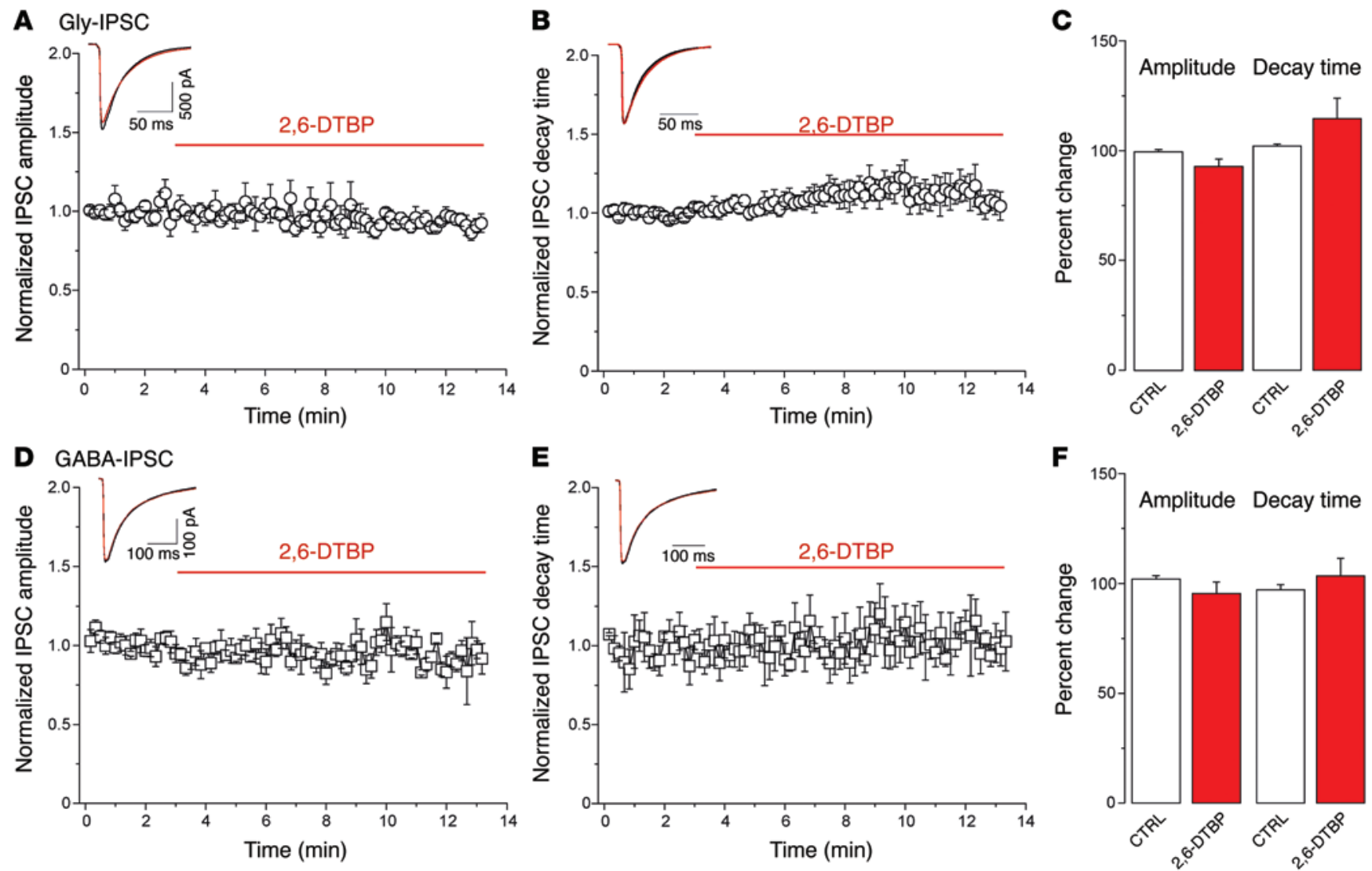

Figure 4. 2,6-DTBP has no significant effects on evoked glycinergic and GABAergic neurotransmission in naive superficial dorsal horn neurons. (A-C) Effects of 2,6-DTBP $(100 \mu \mathrm{M})$ on amplitudes (mean \pm SEM) (A) or decay time constants (B) of light-evoked Gly-IPSCs. Inset, example glycinergic IPSC traces averaged from 10 consecutive current traces (A) or scaled traces (B). Black, control (CTRL) condition; red, 2,6-DTBP. (C) Paired $t$ test, $P=0.12$ and $P=0.28$, for amplitudes and decay time constants, respectively, $n=6$. (D-F) Same as (A-C) but GABA-IPSCs. (F) Paired $t$ test, $P=0.47$ and $P=0.37$ for amplitudes and decay time constants, respectively, $n=6$.

of the homomeric $\alpha 3$ GlyR to glycine (Figure $2 \mathrm{~A}$ ), but significantly impaired sensitivity to 2,6-DTBP. At $100 \mu \mathrm{M}, 2$,6-DTBP potentiated the wild-type $\alpha 3$ GlyR by $164 \% \pm 20 \%(n=12)$, whereas potentiation of the F388A $\alpha 3$ GlyR only reached $10 \% \pm 9.0 \%(n=10)$ (Figure 2, B and C). To gain additional molecular insight, we analyzed the effects of 2,6-DTBP in single-channel recordings of the $\alpha 3$ GlyR carrying the F388A mutation. 2,6-DTBP $(10 \mu \mathrm{M})$ did not significantly modify the activity of F388A mutant ion channels (control $\mathrm{nPo}=0.18 \pm 0.05$ versus 2,6-DTBP $\mathrm{nPo}=0.19 \pm 0.04$, paired $t$ test), confirming the low sensitivity of the mutated receptor to modulation by 2,6-DTBP (wild-type $=192.8 \% \pm 32.6 \%$ of $\mathrm{nPo}$ increase above control versus $\mathrm{F} 388 \mathrm{~A}=5.7 \% \pm 15.6 \%$, unpaired $t$ test, $P<0.001$, Figure 2, D-F, and Supplemental Table 2). Additional analyses revealed that wild-type and F388A mutant GlyR $\alpha 3$ exhibited similar conductance levels (wild-type $\gamma=91.0 \pm 1.20 \mathrm{pS}$ versus F388A $\gamma=88.2 \pm 1.82 \mathrm{pS}, P=0.44$, unpaired $t$ test). These results confirm that the F388A mutation diminished the sensitivity of $\alpha 3$ GlyRs to 2,6-DTBP without altering the ion channel function.

A molecular site for the actions of 2,6-DTBP on the $\alpha 3 G l y R$. The F388 residue required for modulation by 2,6-DTBP lies within the so-called membrane-associated (MA) stretch of the channel immediately upstream of the transmembrane region 4 (25). This site is close to a consensus site for PKA-dependent phosphorylation of GlyR $\alpha 3$ (serine 346) that has been previously implicated in inflammation-induced inhibition of synaptic GlyR currents in the superficial spinal dorsal horn (ref. 6 and Figure 3A; note that the GlyR $\alpha 1$ subunit lacks this consensus site). In order to provide direct evidence for PKA-dependent phosphorylation of S346 in GlyR $\alpha 3$, we made use of the in situ proximity ligation assay (in situ PLA, ref. 26). Incubation of HEK293T cells transiently transfected with wild-type GlyR $\alpha 3$ subunits and EP2 receptors led to a significant increase in the phosphorylation-dependent PLA signal after exposure to $\mathrm{PGE}_{2}(1 \mu \mathrm{M})$. This increase was absent in point-mutated GlyR $\alpha 3(\mathrm{~S} 346 \mathrm{~A})$ (Figure 3, B and C). To explore a potential interaction between phosphorylation at S346 and $\alpha 3$ GlyR modulation by 2,6-DTBP, we investigated the impact of S346 phosphorylation on the $\alpha 3$ GlyR function and modulation and tested two pointmutated receptors that mimic the phosphorylated (S346E) and nonphosphorylated (S346A) state. Electrophysiological recordings revealed that the phosphorylation state did not affect the sensitivity of homomeric or heteromeric $\alpha 3$ GlyRs to their natural agonist glycine (Supplemental Figure 2 and Supplemental Table 1). Likewise, homomeric GlyRs composed of GlyR $\alpha 3$ (S346A) or GlyR $\alpha 3$ (S346E) did not appreciably differ in their sensitivities to 2,6 -DTBP $(142 \% \pm 23 \%$ in S346A versus $190 \% \pm 42 \%$ in $\mathrm{S} 346 \mathrm{E}$, $100 \mu \mathrm{M}$ 2,6-DTBP, Figure 3, D-F). However, heteromeric GlyRs containing the phosphomimicking S346E mutation were significantly more sensitive to 2,6 -DTBP than those containing the 
A Control conditions
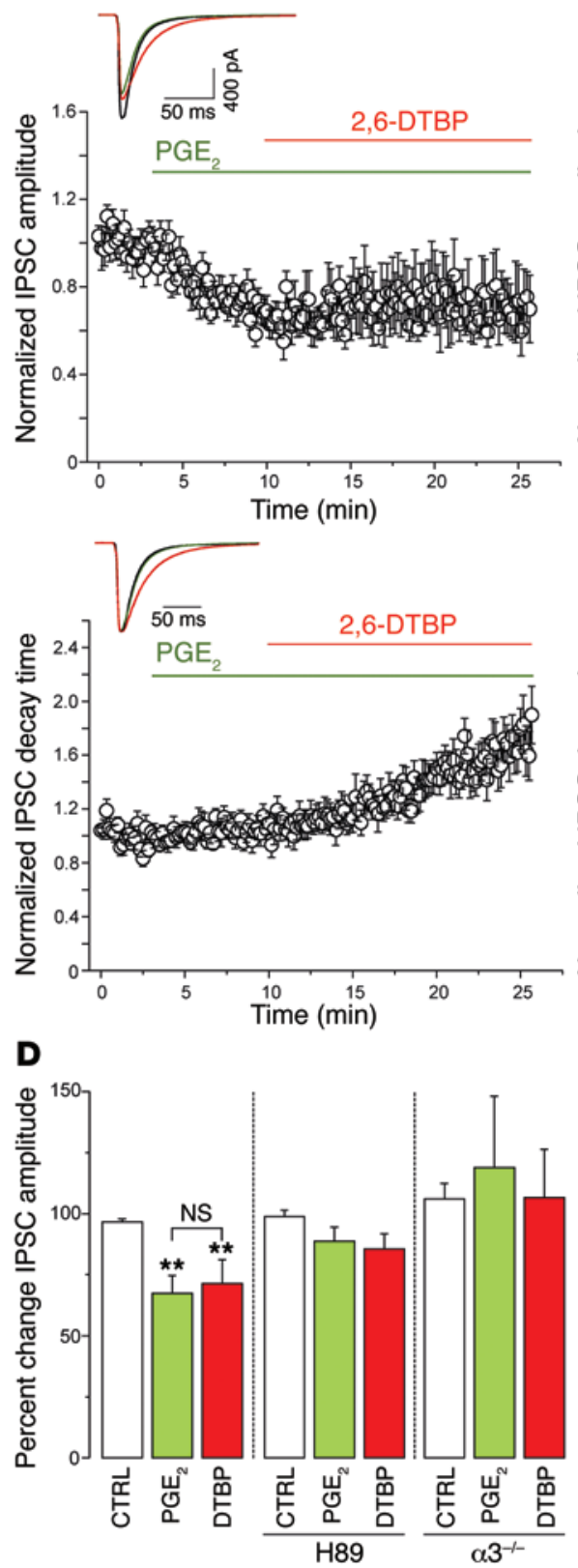

B PKA blockade
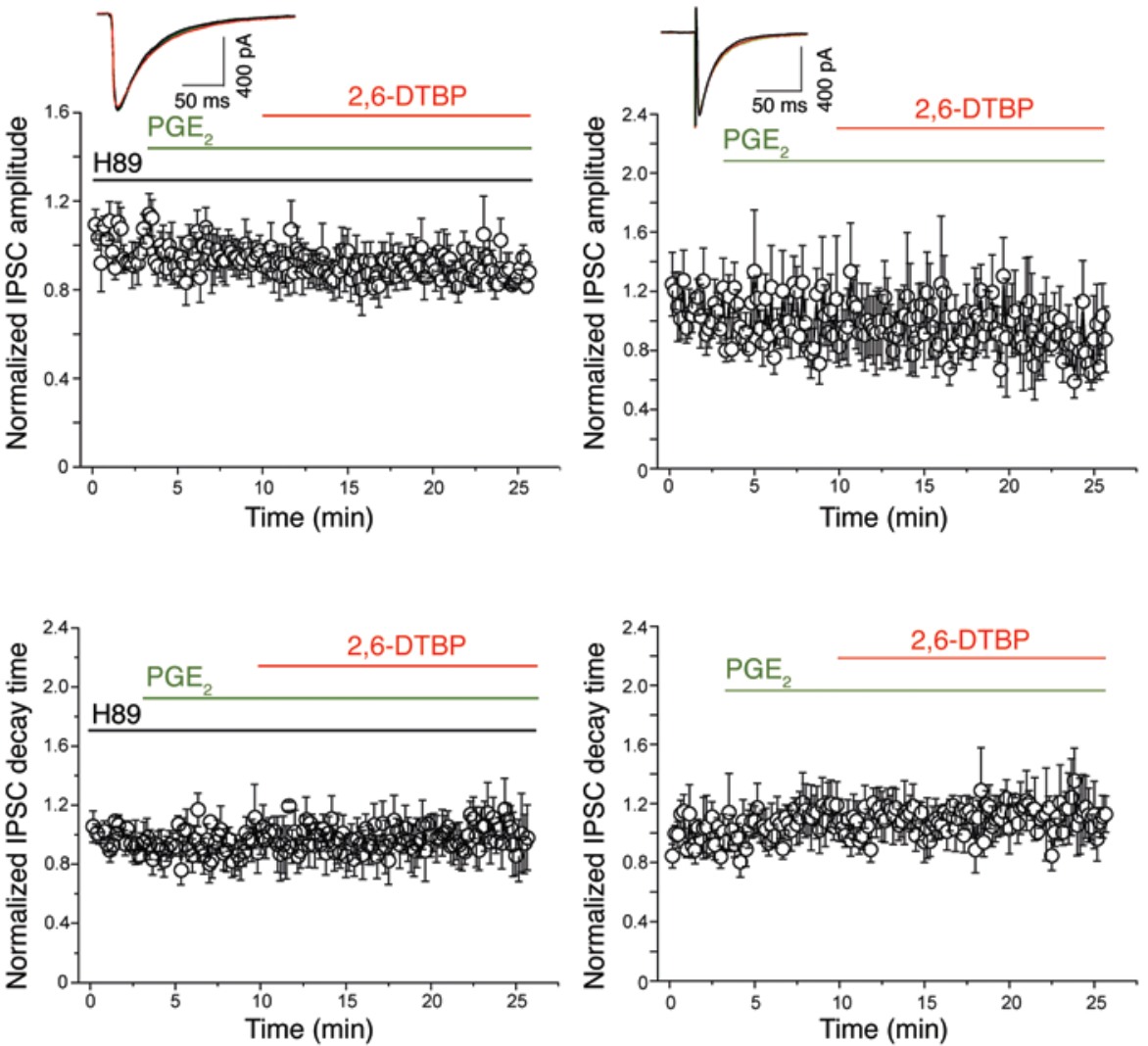

C GlyRo $3^{--1}$

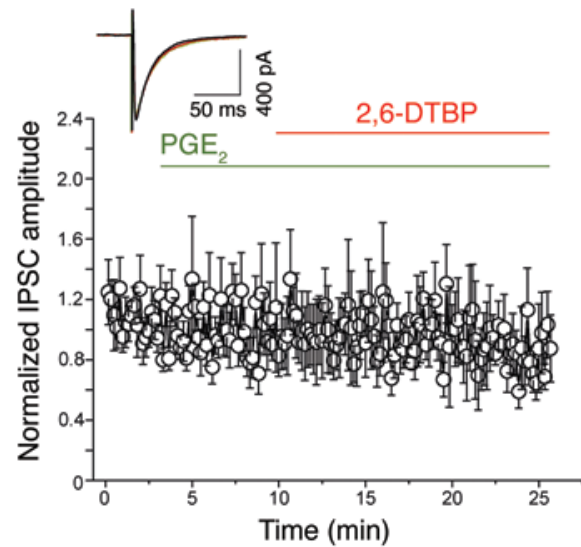

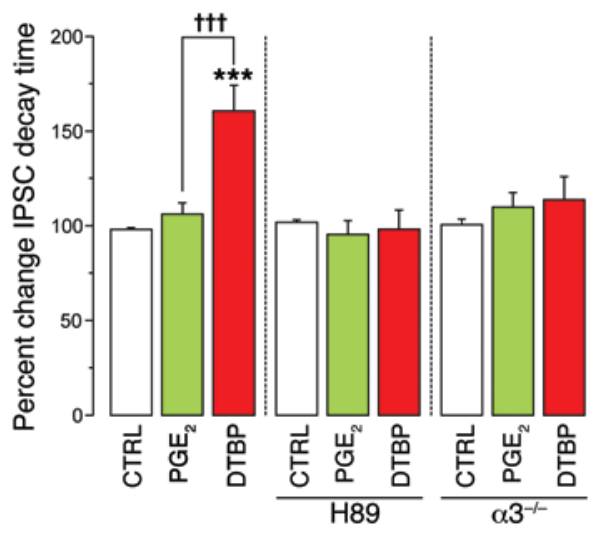

Figure 5. Pretreatment with $\mathbf{P G E}_{2}$ renders synaptic $\mathbf{\alpha 3 \beta G l y R s ~ s u s c e p t i b l e ~ t o ~ m o d u l a t i o n ~ b y ~ 2 , 6 - D T B P . ~ ( A ) ~ N o r m a l i z e d ~ a m p l i t u d e s ~ ( m e a n ~} \pm$ SEM) (top panel) and decay time constants (bottom panel) of light-evoked Gly-IPSC traces versus time, before and during the application of PGE $(1 \mu \mathrm{M})$ and in the additional presence of 2,6-DTBP $(100 \mu \mathrm{M})$. Insets are example traces averaged from 10 consecutive stimulations under the three conditions $(n=12)$. (B) Same as (A) but preincubated with H-89 $(5 \mu \mathrm{M})$ to prevent phosphorylation of GlyRs by $\mathrm{PGE}_{2}(n=7)$. (C) Same as (A) but experiments done in slices taken from GlyR $\alpha 3^{-/-}$mice. In these experiments, Gly-IPSCs were evoked by electrical field stimulation, $n=12$. (D) Left panel: PGE ${ }_{2}$ reduced Gly-IPSC amplitudes in control slices but not in H89-treated slices or in slices from GlyR $\alpha 3^{-1-}$ mice, while 2,6-DTBP had no significant effects on Gly-IPSC amplitudes in either condition. ${ }^{* *} P=0.01$, significant versus control (CTRL). ANOVA followed by Bonferroni post-hoc test. $F(2,35)=8.58, F(2,18)=1.76$, and $F(2,30)=0.12$ for Gly-IPSC amplitudes shown in A-C, respectively. Right panel: 2,6-DTBP significantly prolonged Gly-IPSC decay time courses in PGE ${ }_{2}-$ treated wild-type slices, but not in slices pretreated with $\mathrm{H} 89$, or in slices prepared from GlyR $\alpha 3^{-/-}$mice. ANOVA followed by Bonferroni post-hoc test. ${ }^{\dagger+t} P<0.001$, significant versus $P G E_{2}$; ${ }^{* *} P<0.001$ significant versus control. $F(2,35)=15.66, F(2,18)=0.20$, and $F(2,30)=0.62$ for experiments shown in A-C, respectively. $n=7-12$ cells per group. All scale bars: $50 \mathrm{~ms}, 400 \mathrm{pA}$.

S346A mutation (S346A: $10 \% \pm 8 \%$ versus $\mathrm{S} 346 \mathrm{E}$ : $112 \% \pm 31 \%$, $100 \mu \mathrm{M}$ 2,6-DTBP, $P<0.01$, unpaired $t$ test). These results demonstrate that the heteromeric phosphorylated $\alpha 3 \beta$ GlyRs are more sensitive to modulation by 2,6 -DTBP, indicating a conformationselective modulation of $\alpha 3 \beta$ GlyRs. We then examined whether the mutation of F388 could affect the 2,6-DTBP sensitivity of phosphorylated $\alpha 3$ GlyR. To this end, we studied double point-mutated a3GlyRs containing both the S346E and the F388A mutation. The F388A mutation significantly attenuated the potentiation of homomeric $\alpha 3$ (S346E) GlyRs and heteromeric $\alpha 3$ (S346E) $\beta$ GlyRs 

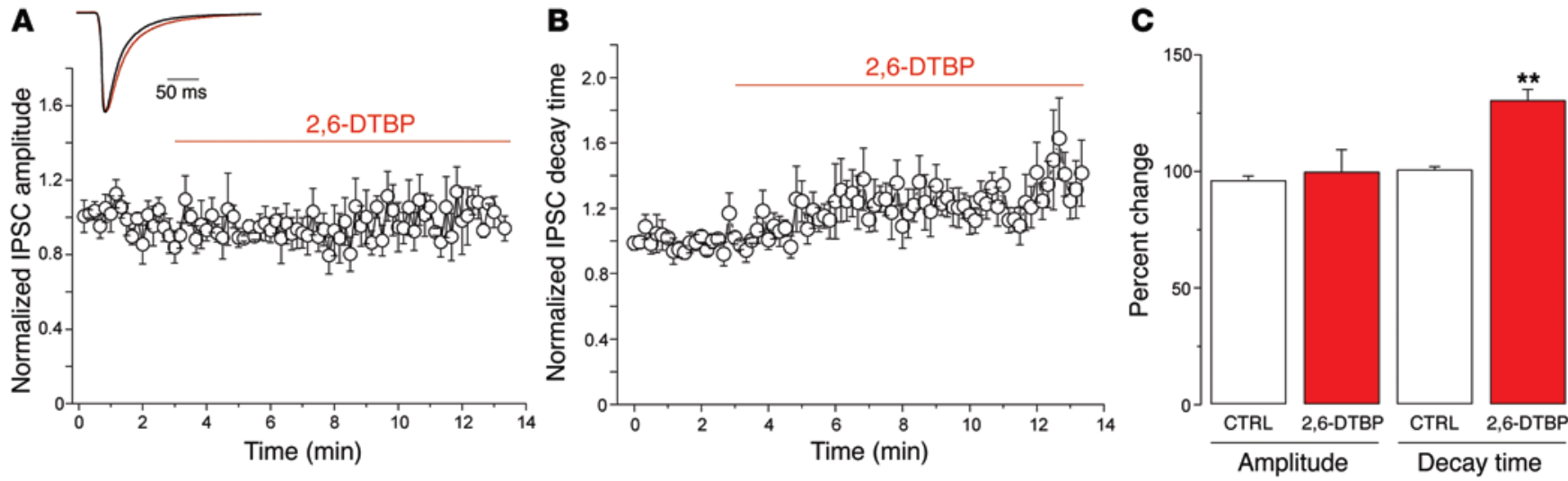

Figure 6. Effects of 2,6-DTBP on the amplitude and decay time of Gly-IPSCs recorded from lamina II neurons of mice with inflamed hindpaws. Recordings were made from the dorsal horn neurons ipsilateral to the inflamed paw. (A) Normalized Cly-IPSC amplitudes over time of whole-cell recording. (B) Same as (A) but normalized decay time constant. (C) 2,6-DTBP had no significant effect on IPSC amplitudes, but significantly increased the decay time constant. ${ }^{* *} P<0.01$, paired $t$ test, $n=6$ cells.

(Figure 3G). These data indicate that the F388 residue is critical for the potentiation of both homomeric $\alpha 3$ and heteromeric (phosphorylated) $\alpha 3 \beta$ GlyRs.

Given the key role of F388 in the effects of 2,6-DTBP on the $\alpha 3 \mathrm{GlyR}$, we next asked whether this residue could be a critical part of an acceptor site for 2,6-DTBP. To this end, we developed a model of the $\alpha 3$ GlyR based on the crystal structures of GluCl and GLICGlyR $(27,28)$. The results of this modeling were not only consistent with F388 being located in the MA stretch close to transmembrane segment 4 (Figure $3 \mathrm{H}$ ), but also provided insights into the molecular composition of the putative acceptor site for 2,6-DTBP. Three residues (F388, M384, and $\mathrm{P381}$ ) appeared to be particularly relevant for hydrophobic interactions between 2,6-DTBP and $\alpha 3$ GlyRs. The charged residues D382 and R385 complement the binding area. Molecular docking analyses revealed a favorable theoretical energy of interaction with this putative acceptor site $\left(\Delta \mathrm{G}_{\mathrm{bind}}=\right.$ $-42.09 \mathrm{kcal} / \mathrm{mol}$, docking score $=-2.133)$. The energy of interaction and the docking scores of 2,6-DTBP and of other propofol analogs with this molecular site correlate well with the functional modulation of wild-type $\alpha 3$ GlyRs (Supplemental Table 3). Interestingly, our modeling data suggest that the introduction of the F388A mutation causes a significant decrease in these parameters $\left(\Delta G_{\text {bind }}\right.$ $=-29.51 \mathrm{kcal} / \mathrm{mol}$, docking score $=-0.507)$, suggesting a direct relationship between the degree of potentiation of the $\alpha 3$ GlyR by 2,6DTBP and the energy of interaction. Additional molecular modeling and simulations suggest that the GlyR $\beta$ subunit also displayed a lower energy of interaction with 2,6-DTBP $\left(\Delta \mathrm{G}_{\text {bind }}=-27.99 \mathrm{kcal} /\right.$ mol, docking score $=0.194)$, possibly because of the presence of an isoleucine at position 388 (i.e., I443 in the $\beta$ subunit) and a poor structural homology with the $\alpha$ subunits in these particular regions (Supplemental Figure 3). Overall, these results identified F388 as a pivotal residue for the effects of 2,6-DTBP on the $\alpha 3 \mathrm{GlyR}$ and suggest the presence of a 2,6-DTBP acceptor site in the MA stretch of $\alpha 3$ that is not present within the $\beta$ subunit.

Modulation of Gly-IPSCs by 2,6-DTBP in superficial dorsal horn neurons. We next characterized the effects of 2,6-DTBP on native GlyRs in excitatory lamina II dorsal horn neurons, i.e., at a site that serves a pivotal role in pain control and which expresses a high density of $\alpha 3$ GlyRs (6). To this end, we prepared lumbar spinal cord slices from VGAT::ChR2 BAC transgenic mice (29), which permit selective activation of inhibitory neurons through short pulses of blue light (30). In slices taken from these mice, excitatory neurons can be readily identified by the absence of a photocurrent (for details see Methods and ref. 30). Of a total of 256 lamina II dorsal horn neurons, 121 were photocurrent negative and hence presumably excitatory. Wide-field stimulation with blue light elicited inhibitory postsynaptic current (IPSC) responses in all these cells. Bicuculline $(10 \mu \mathrm{M})$ or strychnine $(1 \mu \mathrm{M})$ was used to isolate glycinergic or GABAergic IPSCs (Gly-IPSCs or GABA-IPSCs), respectively. We then analyzed the effect of 2,6-DTBP $(100 \mu \mathrm{M})$ on the amplitudes and decay time constants of light-evoked Gly-IPSCs and GABA-IPSCs (Figure 4). As expected from the experiments with recombinant GlyRs, 2,6-DTBP had no significant effect on Gly-IPSCs under basal conditions. Gly-IPSC amplitudes slightly decreased $8.6 \% \pm 3.1 \%$ relative to control values $(n=8, P=0.09$, paired $t$ test), while rise time and decay time slightly increased $13 \%$ $\pm 9 \%$ and $14 \% \pm 9 \%$, respectively $(P=0.50$ and $P=0.17$, paired $t$ test) (Figure 4, A-C). Likewise, light-evoked GABA-IPSCs were not significantly affected $(-6.1 \% \pm 7.3 \% ; P=0.57$, and $1.1 \% \pm 9.6 \%$; $P=0.92$, for amplitudes and decay time constants, respectively, $n=6$ ) (Figure $4, \mathrm{D}-\mathrm{F}$ ). It is likely that most of the receptors underlying these synaptic currents were heteromeric receptors containing $\alpha 1$ and/or $\alpha 3$ subunits together with $\beta$ subunits (6). To address a potential effect on extrasynaptic receptors, we analyzed whether 2,6-DTBP would modulate tonic GlyR currents. In agreement with previous results (31), these experiments revealed the presence of small-amplitude tonic currents $\left(\mathrm{I}_{\text {tonic }}=3.2 \pm 1.0 \mathrm{pA}\right)$ in only a small fraction of the superficial dorsal horn neurons (4 of 14). These tonic currents were not significantly changed by $100 \mu \mathrm{M} 2,6$-DTBP $(+4.6 \% \pm 7.6 \%$ versus control amplitudes, $P=0.56$, paired $t$ test, Supplemental Figure 4). The present results indicate that, in slices taken from naive mice, neither synaptic nor extrasynaptic GlyRs were susceptible to significant modulation by 2,6-DTBP.

Since our data obtained in recombinant GlyRs have shown that the phosphorylation of the $\alpha 3$ subunit at S346 increases the sensitivity of $\alpha 3 \beta$ GlyRs to 2,6-DTBP (compare Figure 3, D-F), we 
A
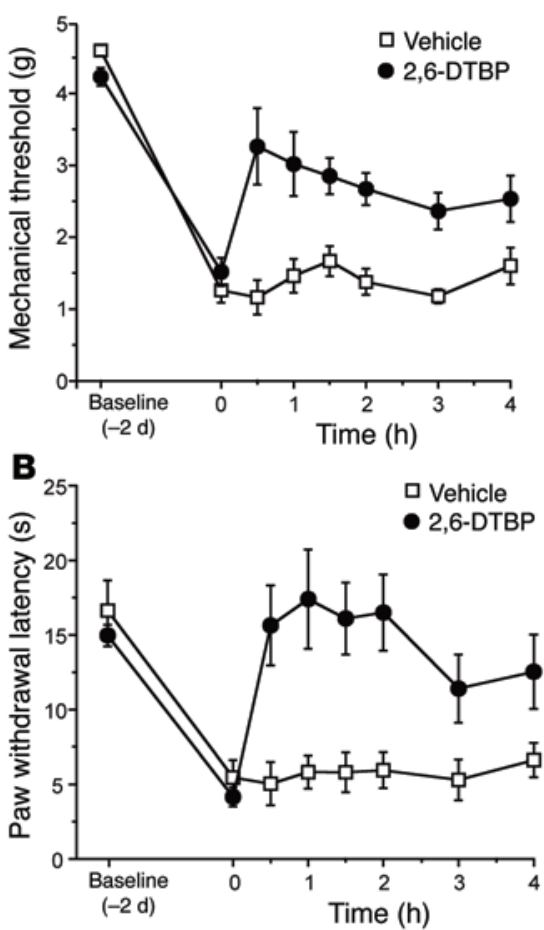

C
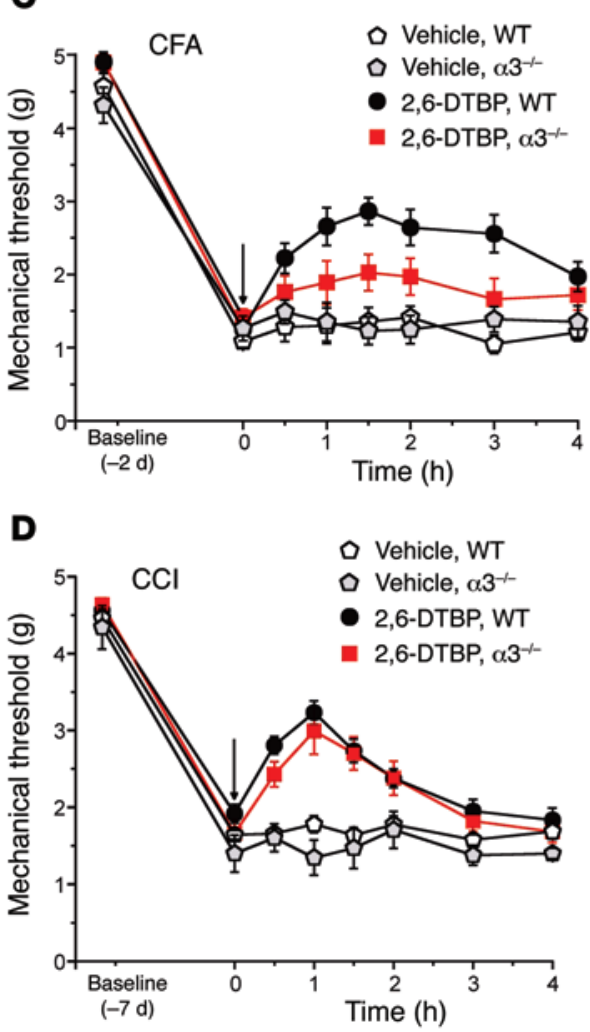
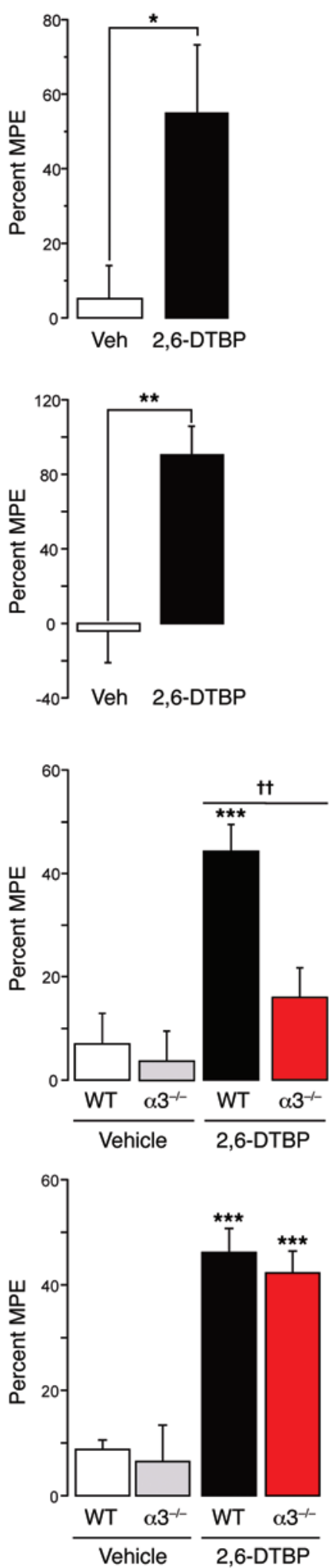

Figure 7. In vivo antihyperalgesic effects of 2,6-DTBP. (A and $\mathbf{B})$ Zymosan A model of inflammatory hyperalgesia. Zymosan A (0.06 mg) was injected subcutaneously into one hindpaw. 2,6-DTBP (90 mg/kg i.p.) or vehicle (VEH) was administered 48 hours after zymosan $A$. (A) Mechanical hyperalgesia. Treatment $\times$ time interactions $(P<0.05)$ were significant for time points between 30 minutes and 2 hours [2-way repeated measures ANOVA, $F(1,6)$ values ranging from 5.0 to 8.2 ]. Right: maximum possible antihyperalgesic effects (MPE) determined for the interval 30 to 60 minutes after 2,6-DTBP injection. ${ }^{*} P<0.05$, unpaired $t$ test, $n=6$ and 7 mice for 2,6-DTPB and vehicle, respectively. (B) Same as (A), but heat hyperalgesia. Treatment $\times$ time interactions were significant for time points between 30 minutes and 4 hours $[F(1,6)=7.2$ to 24.8]. MPEs: Unpaired $t$ test, ${ }^{* *} P<0.01, n=7$ and 6 . (C) Inflammatory hyperalgesia evoked by CFA. 2,6-DTBP was applied 2 days after CFA injection. In wild-type mice ( $n=7$ and 9 ), treatment $\times$ time interactions were significant $(P<0.05)$ for time points between 30 minutes and 4 hours $[F(1,6)=$ 4.8 to 20.0]. In GlyR $\alpha 3^{-/-}$mice ( $n=10$ and 7), interactions remained insignificant for all time points $[P>0.17 ; \mathrm{F}(1,6)$ $=0.02$ to 0.7 ]. Significant MPE of 2,6-DTBP (determined between 60 and 90 minutes) were found in wild-type, but not in GlyR $\alpha 3^{-/-}$mice. The MPE differed significantly between wild-type and GlyR $\alpha 3^{-/-}$mice [ANOVA followed by Bonferroni post-hoc test, $\mathrm{F}(3,38)=9.97 ;{ }^{* * *} P<0.001$, for vehicle versus 2,6-DTBP in wild-type mice]; ${ }^{\dagger+} P<0.01$ (2,6-DTBP effect in wild-type versus GlyR $\alpha 3^{-/-}$mice). (D) Neuropathic hyperalgesia. 2,6-DTBP was applied 7 days after CCl. In wild-type mice, treatment $\times$ time interactions were significant $(P<0.05)$ between 30 minutes and 90 minutes $[F(1,6)=0.09$ to 69.0$]$. In GlyR $\alpha 3^{-1-}$ mice, a significant interaction $(P<0.05)$ was found only for 60 minutes $[F(1,6)=0.003$ to 5.9$]$. The MPE was significant in wild-type and GlyR $\alpha 3^{-/-}$mice [ANOVA followed by Bonferroni post-hoc test, $\mathrm{F}(3,30)=24.7$; ${ }^{* *} P<0.001$, for 2,6 -DTBP versus vehicle]. No significant difference was found between 2,6-DTBP-treated wild-type and GlyR $\alpha 3^{-1-}$ mice $(P=0.55)$. hypothesized that glycinergic synapses containing the $\alpha 3$ subunit might be more sensitive to 2,6-DTBP under inflammatory conditions, i.e., in a phosphorylated state. We therefore assessed the effects of 2,6-DTBP on Gly-IPSCs after preconditioning of the slices with $\mathrm{PGE}_{2}$ (Figure 5). Superfusion of the slices with $\mathrm{PGE}_{2}$ $(1 \mu \mathrm{M})$ significantly reduced the amplitude of the Gly-IPSC (by
$35 \% \pm 5 \%$ relative to control amplitudes, $P<0.001, n=12$, paired $t$ test) but did not change their rise or decay time kinetics (rise time: $-0.2 \% \pm 8 \%$ versus control, decay time: $+6 \% \pm 7 \%$ versus control, $P=0.99$ and $P=0.23$, paired $t$ test, $n=12$ ) (Figure $5, \mathrm{~A}$ and $\mathrm{D}$ ). Subsequent application of 2,6-DTBP significantly increased the decay time constant of Gly-IPSCs by $54 \% \pm 11 \%(P<0.001, n=12$, 
paired $t$ test) but had no significant effect on the amplitudes ( $+8 \%$ $\pm 10 \%$ of amplitudes during $\mathrm{PGE}_{2}, P=0.37, n=12$, paired $t$ test) (Figure 5, A and D). Analyses of the charge transfer occurring during Gly-IPSCs revealed that the prolongation of the decay time by 2,6-DTBP fully compensated for the reduction of Gly-IPSC amplitudes by $\mathrm{PGE}_{2}$. The change in IPSC charge transfer during $\mathrm{PGE}_{2}$ relative to control was $-33 \% \pm 5 \%(P<0.001$, paired $t$ test, $n=12)$. In the additional presence of $2,6-\mathrm{DTBP}$, the total charge transfer increased to $108 \% \pm 18 \%$ of control $(n=12)$. Superfusion of vehicle ( $0.1 \%$ DMSO) had no effect on either the amplitudes or decay kinetics of Gly-IPSCs $(8.5 \% \pm 7.5 \%, P=0.27$, and $19 \% \pm 12 \%$, $P=0.24$, for amplitudes and decay time constants, respectively, $n=7)$. To verify the critical role of PKA-dependent phosphorylation in the priming of spinal cord slices by $\mathrm{PGE}_{2}$, we preincubated slices with the PKA inhibitor H89 $(5 \mu \mathrm{M})$ (Figure 5, B and D). Under these conditions, $\mathrm{PGE}_{2}$ no longer sensitized GlyR to the potentiating actions of 2,6-DTBP (change in decay time constant by 2,6 -DTBP: $-1.8 \% \pm 10.2 \%, P=0.73$, paired $t$ test, $n=7$ ). To further prove that GlyR $\alpha 3$ was the relevant target of PKA-dependent phosphorylation, we performed experiments in slices taken from GlyR $\alpha 3^{-/-}$mice (Figure 5, C and D). Before that, we tested whether the expression of GlyR $\alpha 1$ or GlyR $\beta$ subunits changed following the loss of GlyR $\alpha 3$ expression. Real-time PCR analyses of lumbar spinal cord dorsal horn tissue from wild-type and GlyR $\alpha 3^{-/-}$mice did not reveal significant changes. mRNA copy numbers relative to $\beta$-actin were $0.037 \pm 0.017$ versus $0.047 \pm 0.012$ for the $\alpha 1$ subunit (Glra1; $n=6$ for both groups, $P=0.28$, unpaired $t$ test), and $0.021 \pm 0.009$ versus $0.021 \pm 0.004$ for the $\beta$ subunit (Glrb; $n=6$ for both groups, $P=0.99$, unpaired $t$ test) in wild-type versus $\mathrm{GlyRa}^{-1-}$ mice, respectively. These data are consistent with unchanged expression and distribution of the GlyR $\alpha 1$ protein in the dorsal horn of GlyR $\alpha 3^{--}$mice reported previously (Supplemental Figure 1 in ref. 6). Since the VGAT::ChR2 transgene had not been crossed into GlyR $\alpha 3^{-/-}$mice, we evoked glycinergic IPSCs in these experiments by electrical field stimulation rather than through optogenetic stimulation. In $G l y R \alpha 3^{--}$mice, $\mathrm{PGE}_{2}$ failed to render Gly-IPSCs susceptible to modulation by 2,6-DTBP (change in decay time constant induced by 2,6 -DTBP: $1.2 \% \pm 8.1 \%$ versus control, $P=0.74$, paired $t$ test, $n=12$ ). Because the $G l y R \alpha 3^{-/-}$mice lacked the VGAT::ChR2 transgene, the presence or absence of photocurrents could not be used to distinguish between inhibitory and excitatory neurons. Nevertheless, we expect that at least half of these recordings were made from excitatory neurons (see also ref. 32). Taken together, these results suggest that both PKA inhibition and the absence of GlyR $\alpha 3$ prevented the priming effect of $\mathrm{PGE}_{2}$ on GlyRs, supporting that 2,6-DTBP potentiates synaptic GlyRs in a phosphorylation state-dependent manner.

In order to show that this priming also occurred in vivo in response to peripheral inflammation, we studied the effects of 2,6-DTBP on Gly-IPSCs in slices prepared from five mice with an inflamed hindpaw (Figure 6). Inflammation was induced by subcutaneous injection of zymosan A into the left hindpaw (33), and the development of inflammatory hyperalgesia was confirmed by monitoring mechanical response thresholds. Application of 2,6-DTBP did not significantly alter the Gly-IPSC amplitudes $(+3.6 \% \pm 9.7 \%$ versus control amplitudes, $P=0.72$, paired $t$ test) or rise times $(+13.5 \%$ $\pm 3.8 \%, P=0.09$, paired $t$ test). However, it significantly increased decay time kinetics by $23.5 \% \pm 7.5 \%(P<0.01$, paired $t$ test). Application of vehicle instead of 2,6-DTBP did not significantly change GlyIPSC amplitudes or decay time constants (data not shown).

Antihyperalgesic activity of 2,6-DTBP in behavioral models of pain. Finally, we investigated whether the modulatory effects of 2,6-DTBP on GlyRs translate to antihyperalgesic activity in inflammatory pain in vivo. To this end, we again induced inflammatory hyperalgesia in mice through subcutaneous injection of zymosan A and tested the effects of 2,6-DTBP on mechanical and heat hyperalgesia 48 hours after zymosan A injection (i.e., when central sensitization had reached its maximum; ref. 34). 2,6DTBP significantly reduced mechanical and heat hyperalgesia with a maximum response occurring 30 to 60 minutes after drug administration (Figure 7, A and B). 2,6-DTBP reversed mechanical and heat hyperalgesia at all the time points examined $(P<0.05$, 2-way repeated-measures ANOVA), whereas vehicle did not elicit significant effects. Consistent with our finding that phosphorylation was required to render GlyRs sensitive to 2,6-DTBP, we found that 2,6-DTBP had no analgesic effects against acute nociceptive stimuli (pin prick) in naive mice (withdrawal responses changed from $89 \% \pm 6 \%$ to $92 \% \pm 3 \%$ 30-90 minutes after 2,6-DTBP, $P=0.48$, paired $t$ test, $n=6$ ) (Supplemental Figure 5A). To exclude possible confounding effects of 2,6-DTBP on locomotor activity, motor coordination, and muscle strength, we tested the effects of 2,6-DTBP on locomotion in an open-field arena, in the accelerating rotarod test and in the horizontal wire test. No significant effects were observed (Supplemental Figure 5, B-D).

To determine the contribution of $\alpha 3$ GlyRs to 2,6-DTBPinduced analgesia, we compared the antihyperalgesic effects of 2,6-DTBP in wild-type and Gly $R \alpha 3^{-/-}$mice in different pain models (Figure 7, C and D). We first address inflammatory hyperalgesia. Because previous studies have shown that $G l y R \alpha 3^{-/-}$mice recover quickly from zymosan A-induced hypersensitivity (6), we switched to Complete Freund's Adjuvant (CFA), which causes more prolonged hyperalgesia, and tested the effects of 2,6-DTBP at an early time point (48 hours) after CFA injection. Wild-type and GlyR $\alpha 3^{-/-}$ mice did not significantly differ in their mechanical paw-withdrawal thresholds (PWTs) at this time point and at baseline (Figure 7C). However, heat hyperalgesia in inflamed $\mathrm{Gly} R \alpha 3^{--}$mice was much less pronounced than in wild-type mice, precluding a quantitative comparison of antihyperalgesia by 2,6-DTBP in inflamed wild-type and GlyRa3 $3^{--}$mice (Supplemental Figure 6). We therefore focused on mechanical hyperalgesia, which is also more relevant to chronic pain in human patients (35). 2,6-DTBP significantly reduced mechanical hyperalgesia in wild-type mice by $44.3 \% \pm 5.2 \%$ (Figure 7C). In $G l y R \alpha 3^{--}$mice, this effect was reduced to $16.0 \% \pm 6.2 \%$, indicating that about two thirds of the 2,6-DTBP-evoked analgesia came from an interaction with $\alpha 3$ GlyRs.

Previous work from our group suggested that phosphorylation and inhibition of GlyR $\alpha 3$ contributes to inflammatory but not to neuropathic hyperalgesia (36), while others have shown that 2,6DTBP still alleviated neuropathic hyperalgesia (37). We therefore investigated whether the antihyperalgesic effects of 2,6-DTBP in neuropathic pain also depended on GlyR $\alpha 3$. To this end, we examined the effects of 2,6-DTBP on neuropathic hyperalgesia in wild-type and GlyR $\alpha 3^{-/-}$mice. Neuropathic hyperalgesia was induced by applying a chronic constriction injury (CCI) of the 
left sciatic nerve (38). 2,6-DTBP reduced hyperalgesia in both wild-type and GlyR $3^{---}$mice to very similar degrees (Figure 7D, see also Supplemental Figure 7). The maximum antihyperalgesic responses, which were reached between 30 and 60 minutes after the drug administration, were not significantly different between wild-type and GlyR $\alpha 3^{-/-}$mice (wild-type: $46.1 \% \pm 4.6 \%$ versus GlyR $23^{-1-}: 41.0 \% \pm 4.7 \%$ of maximum possible effect [MPE]), indicating that antihyperalgesic actions against neuropathic pain occurred independently of GlyR $\alpha 3$. Taken together, these findings indicate that dorsal horn GlyRs are modulated by 2,6-DTBP in a phosphorylation state-dependent manner and that this modulation is particularly relevant for inflammatory hyperalgesia. 2,6DTBP also exerts antihyperalgesia against neuropathic pain, but these actions occurred through targets different from $\alpha 3$ GlyRs, possibly involving other GlyRs (16) or hyperpolarization-activated cyclic nucleotide-gated (HCN) channels (37).

\section{Discussion}

Our results establish the nonanesthetic propofol derivative 2,6DTBP as an efficacious enhancer of glycinergic inhibition in the superficial spinal dorsal horn, a key area for central pain control. 2,6-DTBP not only spared GABA $\mathrm{As}$, but also was particularly effective at $\alpha 3$-containing GlyRs, which are abundant in the spinal termination area of nociceptive nerve fibers. While homomeric $\alpha 3 \mathrm{GlyRs}$ were potentiated by 2,6-DTBP under basal conditions, heteromeric ( $\beta$ subunit-containing) GlyRs, which prevail at postsynaptic sites, were potentiated only in a phosphorylated (primed) state. In line with these results, 2,6-DTBP potentiated synaptic GlyR receptor currents in spinal cord slices only after priming with the inflammatory mediator $\mathrm{PGE}_{2}$, or in slices that were obtained from mice with peripheral inflammation. The phosphorylation site responsible for this priming effect is present in $\alpha 3$ GlyRs but is absent in $\alpha 1$ GlyRs.

Mechanisms and sites for modulation. When homomeric GlyR channels expressed in HEK293T cells were investigated, 2,6DTBP shifted the glycine concentration response curve to the left. This leftward shift corresponds well with the increase in channelopen probability observed in our single-channel recording experiments. In spinal cord slices primed with $\mathrm{PGE}_{2}, 2,6-\mathrm{DTBP}$ prolonged the decay of synaptic GlyR currents without altering their amplitudes, consistent with the observed shift in the glycine concentration-response curve. In terms of gross glycinergic inhibition (measured as total charge transfer), this prolongation of the decay time course at least partially counteracted the reduction in the amplitude of glycinergic responses seen in the presence of $\mathrm{PGE}_{2}$.

Our present results indicate that modulation of $\alpha 3$ GlyRs by 2,6DTBP depends on a single phenylalanine residue (F388), which is also required for the modulation of homomeric glycine receptors by propofol, the parent compound of 2,6-DTBP (21). Up to now, a potential role in this process of the $\beta$ subunit and of posttranslational modifications has remained unclear. In the present study, we show that heteromeric $\alpha 3 \beta$ GlyRs (in their nonphosphorylated state) are much less susceptible to modulation by 2,6-DTBP. This is consistent with the presence of an isoleucine residue instead of phenylalanine at the respective position in the $\beta$ subunit (I443), and may in addition suggest that the presence of the $\beta$ subunit prevents 2,6-DTBP from accessing its binding site in the $\alpha$ subunit (compare Supplemental Figure 3). Crystal structures for TM3-TM4 intracel- lular regions and for heteromeric Cys-loop ion channels are still lacking. However, our homology modeling data (Figure 3H) suggest that F388 lies in the so-called MA stretch, i.e., in a region that can influence some biophysical properties of ion channels (25). A comparison of the models of the $\alpha 3-\alpha 3-\alpha 3$ and $\alpha 3-\beta-\alpha 3$ interfaces suggests that the volume of the $\beta$ subunit MA stretch is considerably larger than that of the $\alpha 3$ subunit (by approximately $14,500 \AA^{3}$, Supplemental Figure 8). Together with the functional data, these analyses suggest that, in homomeric $\alpha 3$ GlyRs, all five potential binding sites are accessible to 2,6-DTBP, whereas the presence of $\beta$ subunits in the receptor complex decreases not only the number of acceptor sites but also renders the acceptor sites within the $\alpha$ subunits less accessible to 2,6-DTBP (at least as long as the receptors are in the nonphosphorylated state). An unexpected finding was that the susceptibility of modulation by 2,6-DTBP of heteromeric $\alpha 3 \beta$ GlyRs was restored by phosphorylation of (or introduction of phosphomimetic mutations in) the $\alpha 3$ subunit at S346. This observation suggests a phosphorylation-induced conformational change in the $\alpha 3$ subunit permitting modulation of $\alpha 3 \beta$ GlyRs by 2,6-DTBP (Supplemental Figure 9).

Does modulation of GlyRs contribute to analgesic actions of 2,6-DTBP? 2,6-DTBP not only potentiates GlyRs but also inhibits hyperpolarization and HCN ion channels (37), in particular HCN1 channels, which are expressed in peripheral nociceptors and contribute to pain sensitization (39). In addition, antioxidant properties of 2,6-DTBP $(40,41)$ may directly or indirectly (e.g., via inhibition of the T-type $\mathrm{Ca}^{2+}$ channel, ref. 42) contribute to antihyperalgesia. Our finding that the analgesic effects of 2,6-DTBP in mice with inflamed paws were reduced by about two thirds in Gly $\mathrm{R}^{-1-}$ mice indicates that $\alpha 3$ GlyRs contribute significantly to the analgesic action of 2,6-DTBP. In line with a previous report (37), we found that 2,6-DTBP was active not only against inflammatory pain but also against neuropathic pain caused by peripheral nerve damage. Analgesia against the latter was, however, not diminished in Gly $R \alpha 3^{-/-}$mice, consistent with our previous data that suggested that phosphorylation of $\alpha 3$ GlyRs does not contribute to neuropathic hyperalgesia (36).

In addition to strychnine-sensitive GlyRs, spinal GABA $_{\mathrm{A}} \mathrm{Rs}$ constitute another target that might allow restoring proper synaptic inhibition in the spinal cord during chronic pain states. GABA $\mathrm{A}_{\mathrm{A}}$ offer plenty of opportunities for pharmacological interventions, but currently available drugs targeting $\mathrm{GABA}_{\mathrm{A}}$ Rs do not exert clinically relevant analgesia, mainly because of dose-limiting sedative effects (43). Targeting GlyRs offers the advantage that glycinergic innervation is largely restricted to the hindbrain and spinal cord, and almost completely spares the forebrain, where most of the unwanted actions of GABAergic drugs originate. Consistent with this concept, 2,6-DTBP apparently lacks effects on locomotor activity, motor coordination, and muscle strength, which are typical side effects of classical GABAergic drugs such as the benzodiazepines. It is likely that both the low abundance of glycinergic innervation in the forebrain and specific modulation of phosphorylated GlyRs contribute to this favorable profile.

2,6-DTBP not only showed a preference for GlyRs over $\mathrm{GABA}_{\mathrm{A}}$ Rs but also preferred the low-abundance $\alpha 3$ GlyRs over the much more prevalent $\alpha 1$ GlyRs; the other two GlyR isoforms ( $\alpha 2$ and $\alpha 4$ GlyRs) are less relevant as drug targets. $\alpha 2$ GlyRs are 
mainly expressed during prenatal and early postnatal development (7), and the gene encoding the $\alpha 4$ subunit is a pseudogene in humans $(9,44)$. Compared with $\alpha 1$ GlyRs, which are found throughout most parts of the spinal cord and hindbrain, $\alpha 3$ GlyRs are expressed in a much more spatially restricted manner. In the spinal cord, GlyR $\alpha 3$ subunit expression is limited to the superficial layers of the dorsal horn (6), while at supraspinal sites, a3GlyR expression is generally weak and found only in a few sites, such the retina (45), cerebellum, and hippocampus (46). The preferential potentiation of $\alpha 3$ GlyRs may thus help avoiding unwanted effects such as strong muscle relaxation.

An additional level of specificity comes from the dependence of modulation of synaptic GlyRs on prior phosphorylation. Most postsynaptic GlyRs are heteromers containing, in addition to $\alpha$ subunits, $\beta$ subunits, which anchor the channel complexes to postsynaptic sites. Our experiments revealed that, under resting (nonphosphorylated) conditions, heteromeric $\alpha 3 \beta$ GlyRs were much less sensitive to modulation by 2,6-DTBP than homomeric receptors. However, introduction of a phosphomimetic amino acid exchange in the $\alpha 3$ subunit of recombinant $\alpha 3 \beta$ GlyRs restored susceptibility to modulation by 2,6-DTBP. Accordingly, native postsynaptic GlyRs of the spinal dorsal horn were only modulated after priming through pretreatment of spinal cord slices with $\mathrm{PGE}_{2}$, which leads to PKA-dependent phosphorylation of $\alpha 3 \mathrm{GlyRs}$, or, when slices were taken from mice with an inflamed hindpaw. Importantly, the consensus site for PKA-dependent phosphorylation of GlyR $\alpha 3$ subunits is missing from the $\alpha 1$ subunit, introducing again an additional level of specificity. The absence of this site in GlyR $\alpha 1$ subunits may explain why we did not find effects of 2,6DTBP on muscle strength, even though GlyRs effectively control motoneuron excitability.

Recordings of synaptic GlyR currents in slices obtained from naive (noninflamed) mice were not modulated by 2,6-DTBP, suggesting that homomeric $\alpha 3$ GlyRs lacking GlyR $\beta$ subunits do not make a measurable contribution to synaptic inhibition in the dorsal horn. However, homomeric receptors may be present at extrasynaptic sites where they would mediate tonic currents. In line with a previous report (31), we found tonic currents only in a small portion of cells, and these currents were of very small $(\leq 5$ pA) amplitudes. In certain areas of the brainstem, homomeric $\alpha 1$ GlyRs have been reported to be present in presynaptic terminals where they control transmitter release $(47,48)$. The lack of modulation of glycinergic synaptic currents by 2,6-DTBP suggests that such homomeric presynaptic GlyRs are not present in the superficial dorsal horn. However, it is possible that such receptors exist at other sites and that interaction with these receptors might cause effects of 2,6-DTBP on behaviors of mice not studied here.

In summary, our results describe a phosphorylation statedependent interaction of a propofol derivative with inflammationprimed synaptic GlyRs in the spinal dorsal horn. Reduced analgesic effects in GlyR $\alpha 3^{-/-}$mice suggest that this phosphorylation-dependent potentiation contributes to the analgesic effects of 2,6-DTBP against inflammatory pain (Supplemental Figure 10). These results provide direct evidence that diminished inhibitory pain control in the spinal cord can be restored by positive allosteric modulation of GlyRs, and may pave the path for the generation of new spinally acting analgesics with limited supraspinal side effects.

\section{Methods}

Reagents. 2,6-DTBP and PTX were obtained from Sigma-Aldrich. $\mathrm{PGE}_{2}$, bicuculline, strychnine, QX-314, and H89-dihydrochloride were purchased from Tocris. D-APV and CNQX were obtained from ANAWA. 2,6DTBP and $\mathrm{PGE}_{2}$ were dissolved in DMSO at $100 \mu \mathrm{M}$ and kept at $-20^{\circ} \mathrm{C}$.

Electrophysiology on recombinant GlyRs. Recombinant GlyRs were transiently expressed in HEK293T cells (originally obtained from ATCC-LGC Standards GmbH) using Lipofectamine LTX (Invitrogen). One microgram of DNA per 35-mm dish was used for transfection of $\alpha 1$ and $\alpha 3$ subunits together with $0.5 \mu \mathrm{g}$ of an EGFP expression plasmid to allow identification of the transfected cells. In experiments on $\alpha \beta$ heteromeric GlyRs, plasmids encoding $\alpha$ and $\beta$ subunits were transfected at a ratio of 1:15. To confirm the expression of heteromeric GlyRs, PTX sensitivity was routinely monitored (ref. 7 and Supplemental Figure 1). All recordings were made 18 to 36 hours after transfection. The cDNAs encoding the GlyRs have been described previously (49). Mutations were inserted using the QuickChange site-directed mutagenesis kit (Agilent Technologies) or by an external supplier (Mutagenex). Proper sequences of all constructs were confirmed by full-length sequencing.

Glycine-evoked whole-cell currents were recorded at room temperature $\left(20^{\circ} \mathrm{C}-24^{\circ} \mathrm{C}\right)$ at a holding potential of $-60 \mathrm{mV}$ using the patchclamp technique. Patch electrodes (3-4 M $\Omega$ ) were pulled from borosilicate glass and filled with (in mM): $120 \mathrm{CsCl}, 10$ EGTA, $10 \mathrm{HEPES} \mathrm{(pH}$ 7.4), $4 \mathrm{MgCl}_{2}, 0.5 \mathrm{GTP}$, and $2 \mathrm{ATP}$. The external solution contained (in $\mathrm{mM}) 150 \mathrm{NaCl}, 10 \mathrm{KCl}, 2.0 \mathrm{CaCl}_{2}, 1.0 \mathrm{MgCl}_{2}, 10 \mathrm{HEPES}$ (pH 7.4), and 10 glucose. Recordings were performed with a HEKA EPC-7 amplifier and Patch Master v2.11 software (HEKA Elektronik). Glycine was applied via a manually controlled gravity-fed application system with an inner diameter of $200 \mu \mathrm{m}$ positioned 50 to $120 \mu \mathrm{m}$ from the recorded cell. Glycine $\mathrm{EC}_{10}$ values for each GlyR studied were obtained experimentally after successive application of 1, 10, 30, 60, 100, 200, 500, and $1,000 \mu \mathrm{M}$ glycine (see also Supplemental Table 1 ). The concentration-response curve parameters $\left(\mathrm{EC}_{50}\right.$ and Hill coefficient $[\mathrm{nH}]$ ) were obtained from the curve fits of normalized concentration-response to the equation $\mathrm{I}_{\mathrm{gly}}=\mathrm{I}_{\max }[\mathrm{gly}]^{\mathrm{nH}} /\left([\mathrm{gly}]^{\mathrm{nH}}+\left[\mathrm{EC}_{50}\right]^{\mathrm{nH}}\right)$. The mean maximal current $\left(\mathrm{I}_{\max }\right)$ was determined by applying $1 \mathrm{mM}$ glycine. 2,6-DTBP was first dissolved in DMSO (100\%) at a concentration of $100 \mathrm{mM}$ and subsequently diluted in several steps to the final concentration on the day of the experiment. Before use, solutions were vigorously shaken for 60 minutes. The maximum DMSO concentration was $0.3 \%$. 2,6DTBP was co-applied with glycine, without pre-application. Singlechannel recordings from transfected cells were done as described (19, 28). All recordings were performed in the cell-attached configuration at $19^{\circ} \mathrm{C}-21^{\circ} \mathrm{C}$ with thick-walled borosilicate glass pipettes (final resistance of 10-15 M $\Omega$ ). The extracellular solution contained (in $\mathrm{mM}$ ): 20 Na-gluconate, $102.7 \mathrm{NaCl}, 2 \mathrm{KCl}, 2 \mathrm{CaCl}_{2}, 1.2 \mathrm{MgCl}_{2}, 10$ HEPES, 20 TEA-Cl, 15 sucrose, and 14 glucose, $\mathrm{pH}$ 7.4. The pipette solution was filled with the same extracellular solution but containing glycine (100$150 \mu \mathrm{M})$ or glycine plus 2,6-DTBP $(10 \mu \mathrm{M})$. Single-channel currents were recorded with an RK-400 patch-clamp amplifier (Bio-Logic) at a pipette potential of $+60 \mathrm{mV}$. Recordings were prefiltered at $10 \mathrm{kHz}$ and were digitized to a computer using a Digidata 1400 (Molecular Devices) and pClamp 10 (Axon Instruments).

Molecular modeling and docking. Because most of the structural data available on GlyRs do not include the TM3-TM4 intracellular domain, we modeled the $\alpha 3$ subunit as previously described for the $\alpha 1$ GlyR (50) using the ab initio technique with QUARK (51). The full- 
length GlyR $\alpha 3$ subunit was created by homology modeling using Glu$\mathrm{Cl}$ [PDB: 2RIF] (27) as the template in Modeller 9v13 (52). Additional refinement was performed using the $\alpha 1$ GlyR-GLIC structure (28). The final model was obtained after energy minimization with a conjugate gradient protocol in the software MacroModel (version 9.9, Schrödinger, LLC). The resulting helical conformation of the C-terminal region of the TM3-TM4 intracellular domain (MA stretch) of $\alpha 3$ GlyR was similar to the recently resolved structure for the $5-\mathrm{HT}_{3}$ receptor (53) and to the proposed TM3-TM4 intracellular domain of the GlyR $\alpha 1$ subunit (50). Docking of the protein and ligand was performed using the model of the $\alpha 3$ subunit and the 2,6-DTBP structure available in the ZINC database [ID: ZINC01681254] (54). An initial complex was created with Glide (version 5.9, Schrödinger, LLC) using a receptor grid centered on residue F388 of $\alpha 3$ GlyR. Analysis of the interface by the same software, including structural and energetic parameters, generated a docking score (55). Additionally, a second energy calculation, MM-GBSA, was performed using Prime (version 3.2, Schrödinger, LLC) to predict a theoretical $\Delta \mathrm{G}_{\text {bind }}$. Taken together, the docking score and $\Delta \mathrm{G}_{\text {bind }}$ improved the description of the interaction between $\alpha 3$ GlyR and 2,6-DTBP. The $\beta$ subunit was modeled using the $\alpha 1$ GlyR model (50), and interfaces $\alpha 3-\alpha 3$ and $\alpha 3-\beta$ were constructed based on the $\alpha 1$ GlyR pentamer [PDB: 2M6B] (56). The GlyR $\alpha 3$ F388 mutant was created using BioLuminate (version 1.1, Schrödinger, LLC) and consequent dockings were performed under the same conditions as those described above. All images were created using PyMol (version 1.5, DeLano Scientific LLC).

In situ PLA. $\mathrm{PGE}_{2}$-induced phosphorylation of the GlyR $\alpha 3$ subunit was analyzed using the in situ PLA (26). cDNAs encoding GlyR $\alpha 3$ and EP2, or GlyR $\alpha 3(\mathrm{~S} 346 \mathrm{~A})$ and EP2, were transfected into HEK293T cells grown on poly-lysine-coated coverslips using the PEI (polyethylenimine) method. Two days after transfection, cells were incubated in $1 \mu \mathrm{M} \mathrm{PGE}_{2}$ for 30 minutes. After fixation in $4 \%$ paraformaldehyde for 30 minutes, cells were permeabilized in $0.2 \%$ Triton/ PBS. Phosphorylation of $\alpha 3$ GlyR was tested with the in situ PLA using antibodies directed against $\alpha 3$ GlyR (1:50, Millipore, catalog AB15014) and phosphoserine (1:5, Millipore, catalog 05-1000X). The cells were incubated with the primary antibodies overnight and processed for in situ PLA using the Duolink kit (Sigma-Aldrich) as described previously (57). Images (as z-stack with a $0.8-\mu \mathrm{m} z$-interval) were obtained with a Zeiss 510 Meta confocal laser scanning microscope using a Zeiss $100 \times$ plan-fluar objective (N.A. = 1.4). Quantification of in situ PLA signals was performed by counting individual PLA fluorescence spots using Image $(\mathrm{NIH})$. The optical sections of each image stack were summed into one image, median filtered ( $\mathrm{r}=1.0$ pixels), and subjected to automated spot detection. The number of spots was then normalized to the cell area.

$R T$-PCR. Six lumbar spinal cords were rapidly removed from euthanized adult wild-type and GlyR $\alpha 3^{-/-}$mice. mRNA was transcribed into cDNA using the QuantiTect Reverse Transcription Kit (QIAGEN, catalog 205311). Expression levels of GlyR $\alpha 1$ and GlyR $\beta$ subunits relative to $\beta$-actin were determined using commercially available detection assays (Integrated DNA Technologies, catalog number Mm.PT.58.9052470 for Glra1, Mm.PT.58.29236025 for Glrb, and Mm.PT.58.33257376.gs for Actb).

Recordings in spinal cord slices. Transverse spinal cord slices were prepared from 2- to 3-week-old VGAT::ChR2-eYFP mice (29) and GlyR $\alpha 3$-deficient $\left(G l y R \alpha 3^{-/}\right)$mice (6) of either sex. Slices (400 $\mu \mathrm{m}$ thick) were kept in oxygenated $\left(95 \% \mathrm{O}_{2} / 5 \% \mathrm{CO}_{2}\right.$ ) artificial cerebrospinal fluid (aCSF) containing (in mM): $120 \mathrm{NaCl}, 5 \mathrm{HEPES}, 26 \mathrm{NaHCO}_{3}, 1.25$ $\mathrm{NaH}_{2} \mathrm{PO}_{4}, 2.5 \mathrm{KCl}, 2 \mathrm{CaCl}_{2}, 1 \mathrm{MgCl}_{2}$, and 10 glucose $(\mathrm{pH} 7.35)$ at $35^{\circ} \mathrm{C}$ for 1 hour. Slices were then transferred to the recording chamber, which was continuously perfused with oxygenated aCSF at a flow rate of 1.5 to $2.0 \mathrm{ml} / \mathrm{min}$. Superficial dorsal horn neurons were visually identified using infrared gradient contrast equipment. Recordings in neurons obtained from VGAT::ChR2 mice were made from excitatory neurons identified by the absence of a light-induced photocurrent. Recordings in neurons from GlyR $\alpha 3^{-/}$mice were made from randomly chosen lamina II neurons. Whole-cell patch-clamp recordings were made at room temperature at a holding potential of $-60 \mathrm{mV}$ using a HEKA EPC-10 amplifier and PatchMaster v2.11 software (HEKA Elektronik). Patch pipettes (3.5-4.5 $\mathrm{M} \Omega$ ) were filled with internal solution containing: (in $\mathrm{mM}) 120 \mathrm{CsCl}, 10 \mathrm{EGTA}, 4 \mathrm{MgCl}_{2}, 0.5 \mathrm{GTP}, 2 \mathrm{ATP}$, and $10 \mathrm{HEPES}$ (pH 7.30, adjusted with $\mathrm{CsOH})$. QX-314 (5 mM) was added to block voltageactivated $\mathrm{Na}^{+}$currents in the recorded cell. Once the whole-cell mode was established, the cell was allowed to stabilize for 2 to 5 minutes. Light-evoked IPSCs were elicited by whole-field blue light $(473 \mathrm{~nm})$ illumination (4-ms duration) at a frequency of $0.1 \mathrm{~Hz}$. Glycinergic or GABAergic light-evoked IPSCs were isolated using bicuculline (10 $\mu \mathrm{M})$ or strychnine $(1 \mu \mathrm{M})$, respectively. In GlyR $\alpha 3^{-/-}$mice, glycinergic IPSCs were evoked by electrical field stimulation at a frequency of 0.1 $\mathrm{Hz}(100 \mu \mathrm{s}, 3-30 \mathrm{~V})$ using a glass electrode (5-7 M 2 ) filled with standard extracellular solution and placed about $50 \mu \mathrm{m}$ from the recorded neuron (6). Gly-IPSCs were isolated using CNQX (5 $\mu \mathrm{M})$, D-APV (50 $\mu \mathrm{M})$, and bicuculline $(10 \mu \mathrm{M})$. After 3 to 5 minutes of baseline recording, 2,6-DTBP $(100 \mu \mathrm{M})$ or $\mathrm{PGE}_{2}(1 \mu \mathrm{M})$ was added to the bath solution for 8 to 10 minutes. To examine the role of PKA-dependent phosphorylation, slices were continuously superfused with $\mathrm{H} 89(5 \mu \mathrm{M})$. At the end of each recording, strychnine $(1 \mu \mathrm{M})$ or bicuculline $(10 \mu \mathrm{M})$ was added to confirm the glycinergic or GABAergic nature of the recorded IPSCs.

Animals. Breeding pairs of GlyR $\alpha 3^{-/-}$mice (6) were provided by Robert J. Harvey, Ulrike Müller, and Heinrich Betz (all at the Max Planck Institute for Brain Research, Frankfurt, Germany). VGAT::ChR2eYFP mice (29) were provided by Guoping Feng, McGovern Institute for Brain Research at MIT, Cambridge, Massachusetts, USA. GlyR $\alpha 3^{-/-}$ mice and VGAT::ChR2-eYFP BAC transgenic mice were maintained on a C57BL/6 background. All mice were group housed with a standard 12-hour light/dark cycle and food and water available ad libitum.

Behavioral tests. Antihyperalgesic properties of 2,6-DTBP $(90 \mathrm{mg} /$ $\mathrm{kg}$ i.p.) were studied in 7- to 12-week-old mice subjected to the zymosan A and CFA models of inflammatory hyperalgesia $(6,33)$. Mice were injected subcutaneously with either zymosan A (0.06 mg in $20 \mu \mathrm{l}$ saline) or CFA (1:2 diluted in saline) into the plantar side of the left hind paw. To study the effects of 2,6-DTBP on neuropathic pain, mice were subjected to the CCI (38). In brief, three loose (5-0 silk) ligatures were put around the left sciatic nerve proximal to the trifurcation. Mice that showed signs of paralysis or did not develop significant hypersensitivity were excluded from subsequent experiments. Mechanical and thermal nociceptive sensitivities were determined using electronic von Frey filaments and the plantar test, respectively. 2,6-DTBP was dispersed in saline/0.05\% Tween 20 (vehicle), sonicated on ice, and injected i.p. (see also refs. 37 and 58). Sensitivities of the ipsilateral (inflamed or nerve-injured) paw and the contralateral control paw were measured alternately and at least four measurements were taken per mouse for all time points. Antihyperalgesia was quantified for a time interval of 
30 to 60 (for zymosan A model and CCI) or 60 to 90 minutes (for CFA) after drug injection when the drug effect was maximal, and expressed as percentage of maximum possible analgesic effect (MPE). MPE $=\left(\mathrm{R}_{\text {post-drug }}-\mathrm{R}_{\text {pre-drug }}\right) /\left(\mathrm{R}_{\text {baseline }}-\mathrm{R}_{\text {pre-drug }}\right) \times 100$, where $\mathrm{R}$ is the average response latency or threshold under baseline condition $\left(\mathrm{R}_{\text {baseline }}\right)$, after induction of inflammation but before drug injection $\left(\mathrm{R}_{\text {pre-drug }}\right)$, and after drug injection $\left(\mathrm{R}_{\text {post-drug }}\right)$. Accelerating rotarod performance (from 4 to $40 \mathrm{rpm}$ ) of wild-type mice was measured 60 minutes after the administration of 2,6-DTBP or vehicle. Each mouse was tested three times. Mice were trained with 2 different training sessions on 2 consecutive days before testing. The locomotor activity assays were performed during the light phase of the day-night cycle. The animals were placed in individual circular enclosures (20-cm diameter) equipped with 4 photocells. The locomotor activity was expressed as the total number of photocell interruptions recorded in a period of 6 hours immediately after drug administration. The horizontal wire test (59) was performed to assess potential muscle relaxation. Mice were placed forepaws-first onto a $15-\mathrm{cm}$ metal wire and scored according to whether or not they were able to grasp the wire with their hindpaws. Acute pain was measured in the so-called pin-prick test using an injection needle applied to the plantar surface of the hindpaw without breaking the skin. The number of paw withdrawals out of three trials was calculated as a percentage for each time point. The experimenter was blind to mouse genotype and drug treatment. Special care was taken to ensure equal numbers of age-matched male and female mice in all behavioral experiments.

Statistics. All data are displayed as the mean \pm SEM. Statistical comparisons were made with 2-tailed paired or unpaired $t$ tests or with ANOVA or repeated measures ANOVA followed by appropriate post-hoc tests. $P$ values less than 0.05 were considered statistically significant.

Study approval. Permission for and approval of the animal experiments was obtained from the Tierversuchskommission of the canton of Zurich, Zurich, Switzerland (license numbers 135/2009 and 126/2012).

\section{Author contributions}

MAA, GEY, WTR, DB, ADL, COL, BM, and GMC performed experiments and analyzed the data. CFB performed the molecular modeling analyses and calculations. GEY, PJC, and HUZ designed the experiments. MAA, GEY, and HUZ wrote the manuscript. All authors made comments on the manuscript.

\section{Acknowledgments}

We are thankful to Thomas Grampp and Louis Scheurer for excellent technical assistance, to Isabelle Kellenberger for mouse genotyping, and to Carmen Brazerol, Conny Leuzinger, Dennis Kwame, and Ana Hemmersbach for animal caretaking. This work was supported by grants from the Swiss National Science Foundation (SNSF; 131093) and an advanced investigator grant from the European Research Council (ERC AdG DHISP 250128) to H.U. Zeilhofer, by grants from the Fondation de la Recherche Médicale, Institut Pasteur, to G. Moraga-Cid and P.-J. Corringer and the Agence Nationale de la Recherche Grants to P.-J. Corringer, VRIDUniversity of Concepcion 213.033.106-1.0, and FONDECYT 1140515 to G.E. Yévenes. G.E. Yévenes was supported through a Forschungskredit from the University of Zurich. W.T. Ralvenius was supported through a fellowship from the Stiftung für Forschung of the Medical Faculty, University of Zurich.

Address correspondence to: Hanns Ulrich Zeilhofer, Institute of Pharmacology and Toxicology, University of Zurich, Winterthurerstrasse 190, CH-8057 Zürich, Switzerland. Phone: 41.44.63.55.912; E-mail: zeilhofer@pharma.uzh.ch. Or to: Gonzalo E. Yévenes, Department of Physiology, Faculty of Biological Sciences, University of Concepcion, Barrio Universitario S/N, 4070386, Concepcion, Chile. Phone: 56.41.266.13.90; E-mail: gyevenes@udec.cl.
1. Basbaum AI, Bautista DM, Scherrer G, Julius D. Cellular and molecular mechanisms of pain. Cell. 2009;139(2):267-284.

2. Zeilhofer HU, Benke D, Yévenes GE. Chronic pain states: pharmacological strategies to restore diminished inhibitory spinal pain control. Annu Rev Pharmacol Toxicol. 2012;52:111-133.

3. Luo C, Kuner T, Kuner R. Synaptic plasticity in pathological pain. Trends Neurosci. 2014;37(6):343-355.

4. Sandkühler J. Models and mechanisms of hyperalgesia and allodynia. Physiol Rev. 2009;89(2):707-758.

5. Coull JA, et al. Trans-synaptic shift in anion gradient in spinal lamina I neurons as a mechanism of neuropathic pain. Nature. 2003;424(6951):938-942.

6. Harvey RJ, et al. GlyR $\alpha 3$ : an essential target for spinal PGE2-mediated inflammatory pain sensitization. Science. 2004;304(5672):884-887.

7. Lynch JW. Native glycine receptor subtypes and their physiological roles. Neuropharmacology. 2009;56(1):303-309.

8. Yévenes GE, Zeilhofer HU. Allosteric modulation of glycine receptors. Br J Pharmacol. 2011;164(2):224-236.

9. Laube B, Maksay G, Schemm R, Betz H. Modulation of glycine receptor function: a novel approach for therapeutic intervention at inhibitory synapses? Trends Pharmacol Sci. 2002;23(11):519-527.

10. de la Roche J, et al. 4-Chloropropofol enhances chloride currents in human hyperekplexic and artificial mutated glycine receptors. BMC Neurol. 2012;12:104.

11. Eckle VS, et al. 4-bromopropofol decreases action potential generation in spinal neurons by inducing a glycine receptor-mediated tonic conductance. Br J Pharmacol. 2014;171(24):5790-5801.

12. Xiong W, et al. Cannabinoid potentiation of glycine receptors contributes to cannabis-induced analgesia. Nat Chem Biol. 2011;7(5):296-303.

13. Xiong W, et al. Cannabinoids suppress inflammatory and neuropathic pain by targeting $\alpha 3$ glycine receptors. J Exp Med. 2012;209(6):1121-1134.

14. Yang Z, Aubrey KR, Alroy I, Harvey RJ, Vandenberg RJ, Lynch JW. Subunit-specific modulation of glycine receptors by cannabinoids and $\mathrm{N}$-arachidonyl-glycine. Biochem Pharmacol. 2008;76(8):1014-1023.

15. Zhang JY, Gong N, Huang JL, Guo LC, Wang YX. Gelsemine, a principal alkaloid from Gelsemium sempervirens Ait., exhibits potent and specific antinociception in chronic pain by acting at spinal $\alpha 3$ glycine receptors. Pain. 2013;154(11):2452-2462.

16. Ahrens J, et al. 2,6 di-tert-butylphenol, a nonanesthetic propofol analog, modulates $\alpha 1 \beta$ glycine receptor function in a manner distinct from propofol. Anesth Analg. 2004;99(1):91-96.

17. Ahrens J, Leuwer M, de la Roche J, Foadi N, Krampfl K, Haeseler G. The non-anaesthetic propofol analogue 2,6-di-tert-butylphenol fails to modulate $\mathrm{GABA}_{\mathrm{A}}$ receptor function. Pharmacology. 2009;83(2):95-98.

18. Krasowski MD, Jenkins A, Flood P, Kung AY, Hopfinger AJ, Harrison NL. General anesthetic potencies of a series of propofol analogs correlate with potency for potentiation of $\gamma$-aminobutyric acid (GABA) current at the $\mathrm{GABA}_{\mathrm{A}}$ receptor but not with lipid solubility. J Pharmacol Exp Ther. 2001;297(1):338-351.

19. Marabelli A, Moroni M, Lape R, Sivilotti LG. The kinetic properties of the $\alpha 3$ rat glycine receptor make it suitable for mediating fast synaptic inhibition. J Physiol. 2013;591(pt 13):3289-3308.

20. Ahrens J, et al. A transmembrane residue influences the interaction of propofol with the strychnine-sensitive glycine $\alpha 1$ and $\alpha 1 \beta$ receptor. Anesth Analg. 2008;107(6):1875-1883.

21. Moraga-Cid G, Yévenes GE, Schmalzing G, Peoples RW, Aguayo LG. A single phenylalanine residue in the main intracellular loop of $\alpha 1$ $\gamma$-aminobutyric acid type A and glycine receptors influences their sensitivity to propofol. Anesthesiology. 2011;115(3):464-473. 
22. Lynagh T, Laube B. Opposing effects of the anesthetic propofol at pentameric ligand-gated ion channels mediated by a common site. J Neurosci. 2014;34(6):2155-2159.

23. Mihic SJ, et al. Sites of alcohol and volatile anaesthetic action on $\mathrm{GABA}_{\mathrm{A}}$ and glycine receptors. Nature. 1997;389(6649):385-389.

24. Findlay GS, et al. Glycine receptor knock-in mice and hyperekplexia-like phenotypes: comparisons with the null mutant. J Neurosci. 2003;23(22):8051-8059.

25. Carland JE, et al. Characterization of the effects of charged residues in the intracellular loop on ion permeation in $\alpha 1$ glycine receptor channels. J Biol Chem. 2009;284(4):2023-2030.

26. Leuchowius KJ, et al. High content screening for inhibitors of protein interactions and post-translational modifications in primary cells by proximity ligation. Mol Cell Proteomics. 2010;9(1):178-183

27. Hibbs RE, Gouaux E. Principles of activation and permeation in an anion-selective Cys-loop receptor. Nature. 2011;474(7349):54-60.

28. Moraga-Cid G, et al. Allosteric and hyperekplexic mutant phenotypes investigated on an $\alpha 1$ glycine receptor transmembrane structure. Proc Natl Acad Sci U S A. 2015;112(9):2865-2870.

29. Zhao S, et al. Cell type-specific channelrhodop$\sin -2$ transgenic mice for optogenetic dissection of neural circuitry function. Nat Methods. 2011;8(9):745-752

30. Foster E, et al. Targeted ablation, silencing, and activation establish glycinergic dorsal horn neurons as key components of a spinal gate for pain and itch. Neuron. 2015;85(6):1289-1304.

31. Mitchell EA, Gentet LJ, Dempster J, Belelli D. $\mathrm{GABA}_{\mathrm{A}}$ and glycine receptor-mediated transmission in rat lamina II neurones: relevance to the analgesic actions of neuroactive steroids. JPhysiol. 2007;583(pt 3):1021-1040.

32. Todd AJ, Spike RC. The localization of classical transmitters and neuropeptides within neurons in laminae I-III of the mammalian spinal dorsal horn. Prog Neurobiol. 1993;41(5):609-645.

33. Meller ST, Gebhart GF. Intraplantar zymosan as a reliable, quantifiable model of thermal and mechanical hyperalgesia in the rat. Eur J Pain. 1997;1(1):43-52.

34. Reinold $\mathrm{H}$, et al. Spinal inflammatory hyperalgesia is mediated by prostaglandin $\mathrm{E}$ receptors of the EP2 subtype. J Clin Invest. 2005;115(3):673-679.

35. Schaible H-G. Joint pain: basic mechanisms. In:
McMahon SB, Koltzenburg M, Tracey I, Turk DC, eds. Wall and Melzack's Textbook of Pain. 6th ed. Philadelphia, Pennsylvania, USA: Elsevier Saunders; 2013:609-619.

36. Hösl K, Reinold H, Harvey RJ, Müller U, Narumiya S, Zeilhofer HU. Spinal prostaglandin E receptors of the EP2 subtype and the glycine receptor $\alpha 3$ subunit, which mediate central inflammatory hyperalgesia, do not contribute to pain after peripheral nerve injury or formalin injection. Pain. 2006;126(1-3):46-53.

37. Tibbs GR, et al. HCN1 channels as targets for anesthetic and nonanesthetic propofol analogs in the amelioration of mechanical and thermal hyperalgesia in a mouse model of neuropathic pain. J Pharmacol Exp Ther. 2013;345(3):363-373.

38. Bennett GJ, Xie YK. A peripheral mononeuropathy in rat that produces disorders of pain sensation like those seen in man. Pain. 1988;33(1):87-107.

39. Momin A, Cadiou H, Mason A, McNaughton PA. Role of the hyperpolarization-activated current Ih in somatosensory neurons. J Physiol. 2008;586(pt 24):5911-5929.

40. Ku G, Doherty NS, Schmidt LF, Jackson RL, Dinerstein RJ. Ex vivo lipopolysaccharide-induced interleukin-1 secretion from murine peritoneal macrophages inhibited by probucol, a hypocholesterolemic agent with antioxidant properties. FASEB J. 1990;4(6):1645-1653.

41. Shakir RM, Ariffin A, Abdulla MA. Synthesis of new 2,5-di-substituted 1,3,4-oxadiazoles bearing 2,6-di-tert-butylphenol moieties and evaluation of their antioxidant activity. Molecules. 2014;19(3):3436-3449.

42. Nelson MT, et al. Molecular mechanisms of subtype-specific inhibition of neuronal T-type calcium channels by ascorbate. J Neurosci. 2007;27(46):12577-12583.

43. Ralvenius WT, Benke D, Acuña MA, Rudolph U, Zeilhofer HU. Analgesia and unwanted benzodiazepine effects in point-mutated mice expressing only one benzodiazepine-sensitive $\mathrm{GABA}_{\mathrm{A}}$ receptor subtype. Nat Commun. 2015;6:6803.

44. Simon J, Wakimoto H, Fujita N, Lalande M, Barnard EA. Analysis of the set of $\mathrm{GABA}_{\mathrm{A}}$ receptor genes in the human genome. J Biol Chem. 2004;279(40):41422-41435.

45. Haverkamp S, Müller U, Harvey K, Harvey RJ, Betz H, Wässle H. Diversity of glycine receptors in the mouse retina: localization of the $\alpha 3$ subunit. JComp Neurol. 2003;465(4):524-539.

46. Malosio ML, Marqueze-Pouey B, Kuhse J, Betz $\mathrm{H}$. Widespread expression of glycine receptor subunit mRNAs in the adult and developing rat brain. EMBO J. 1991;10(9):2401-2409.

47. Xiong W, et al. Presynaptic glycine receptors as a potential therapeutic target for hyperekplexia disease. Nat Neurosci. 2014;17(2):232-239.

48. Hruskova B, et al. Differential distribution of glycine receptor subtypes at the rat calyx of Held synapse. J Neurosci. 2012;32(47):17012-17024.

49. Yévenes GE, Zeilhofer HU. Molecular sites for the positive allosteric modulation of glycine receptors by endocannabinoids. PLoS One. 2011;6(8):e23886.

50. Burgos CF, Castro PA, Mariqueo T, Bunster M, Guzman L, Aguayo LG. Evidence for $\alpha$-helices in the large intracellular domain mediating modulation of the $\alpha 1$-glycine receptor by ethanol and G $\beta \gamma$. JPharmacol Exp Ther. 2015;352(1):148-155.

51. Xu D, Zhang Y. Ab initio protein structure assembly using continuous structure fragments and optimized knowledge-based force field. Proteins. 2012;80(7):1715-1735.

52. Eswar N, et al. Comparative protein structure modeling using Modeller. Curr Protoc Bioinformatics. 2006; Chapter 5:Unit 5.6.

53. Hassaine G, et al. X-ray structure of the mouse serotonin 5-HT3 receptor. Nature. 2014;512(7514):276-281.

54. Irwin JJ, Sterling T, Mysinger MM, Bolstad ES, Coleman RG. ZINC: a free tool to discover chemistry for biology. JChem Inf Model. 2012;52(7):1757-1768.

55. Friesner RA, et al. Extra precision glide: docking and scoring incorporating a model of hydrophobic enclosure for protein-ligand complexes. JMed Chem. 2006;49(21):6177-6196.

56. Mowrey DD, et al. Open-channel structures of the human glycine receptor $\alpha 1$ fulllength transmembrane domain. Structure. 2013;21(10):1897-1904.

57. Zemoura K, Benke D. Proteasomal degradation of $\gamma$-aminobutyric acid receptors is $_{B}$ mediated by the interaction of the $\mathrm{GABA}_{\mathrm{B} 2} \mathrm{C}$ terminus with the proteasomal ATPase Rtp6 and regulated by neuronal activity. J Biol Chem. 2014;289(11):7738-7746.

58. James R, Glen JB. Synthesis, biological evaluation, and preliminary structure-activity considerations of a series of alkylphenols as intravenous anesthetic agents. JMed Chem. 1980;23(12):1350-1357.

59. Bonetti EP, et al. Benzodiazepine antagonist Ro 15-1788: neurological and behavioral effects. Psychopharmacology (Berl). 1982;78(1):8-18. 